\title{
1-(1-Arylethylpiperidin-4-yl)thymine Analogs as Antimycobacterial TMPK Inhibitors
}

\author{
Yanlin Jian ${ }^{1}$, Fabian Hulpia ${ }^{1}$, Martijn D. P. Risseeuw ${ }^{1}$, He Eun Forbes ${ }^{2}$, Guy Caljon ${ }^{3}$, \\ Hélène Munier-Lehmann ${ }^{4}\left(\mathbb{D}\right.$, Helena I. M. Boshoff ${ }^{2}$ and Serge Van Calenbergh ${ }^{1, * \mathbb{C}}$ \\ 1 Laboratory for Medicinal Chemistry (FFW), Ghent University, Ottergemsesteenweg 460, B-9000 Gent, \\ Belgium; yanlin.jian@ugent.be (Y.J.); fabian.hulpia@ugent.be (F.H.); martijn.risseeuw@ugent.be (M.D.P.R.) \\ 2 Tuberculosis Research Section, Laboratory of Clinical Immunology and Microbiology, National Institute of \\ Allergy and Infectious Disease, National Institutes of Health, 9000 Rockville Pike, Bethesda, MD 20892, USA; \\ grace.chun@nih.gov (H.E.F.); hboshoff@niaid.nih.gov (H.I.M.B.) \\ 3 Laboratory of Microbiology, Parasitology and Hygiene, University of Antwerp, Universiteitsplein 1 (S7), \\ B-2610 Wilrijk, Belgium; Guy.Caljon@uantwerpen.be \\ 4 Unit of Chemistry and Biocatalysis, Department of Structural Biology and Chemistry, Institut Pasteur, \\ CNRS UMR3523, 28 Rue du Dr. Roux, CEDEX 1575724 Paris, France; helene.munier-lehmann@pasteur.fr \\ * Correspondence: serge.vancalenbergh@ugent.be; Tel.: +32-9-264-81-24
}

Academic Editor: Athina Geronikaki

Received: 6 May 2020; Accepted: 14 June 2020; Published: 17 June 2020

\begin{abstract}
A series of Mycobacterium tuberculosis TMPK (MtbTMPK) inhibitors based on a reported compound 3 were synthesized and evaluated for their capacity to inhibit MtbTMPK catalytic activity and the growth of a virulent $M$. tuberculosis strain $(\mathrm{H} 37 \mathrm{Rv})$. Modifications of the scaffold of $\mathbf{3}$ failed to afford substantial improvements in MtbTMPK inhibitory activity and antimycobacterial activity. Optimization of the substitution pattern of the D ring of $\mathbf{3}$ resulted in compound $\mathbf{2 1} \mathbf{j}$ with improved MtbTMPK inhibitory potency (three-fold) and H37Rv growth inhibitory activity (two-fold). Moving the 3-chloro substituent of $\mathbf{2 1} \mathbf{j}$ to the para-position afforded isomer $\mathbf{2 1 h}$, which, despite a 10 -fold increase in $\mathrm{IC}_{50}$-value, displayed promising whole cell activity (minimum inhibitory concentration $(\mathrm{MIC})=12.5 \mu \mathrm{M})$.
\end{abstract}

Keywords: tuberculosis; Mycobacterium tuberculosis; MtbTMPK; 1-(1-arylethylpiperidin-4-yl)thymine

\section{Introduction}

Tuberculosis (TB) is an airborne infectious disease caused by Mycobacterium tuberculosis (M. tuberculosis). Still belonging to the top ten causes of death worldwide, TB was responsible for claiming 1.5 million lives in 2018, thereby preceding AIDS [1]. The World Health Organization (WHO) launched the "END TB" strategy in 2014, aiming at reducing the incidence of TB by $90 \%$ and the number of deaths from TB by $95 \%$ by 2035 compared with 2015 levels [2]. Nevertheless, the progress towards this sustainable development goal is disappointing, and the continuing increase in drug-resistant TB cases makes the situation more challenging $[1,3]$.

Patients with drug-sensitive TB are currently treated with a combination regimen, consisting of a two-month treatment with first-line agents rifampicin, isoniazid, pyrazinamide and ethambutol, followed by a four-month treatment with rifampicin and isoniazid. Although this schedule has decreased TB mortality, these gains are being threatened by the advent of coinfection with HIV/AIDS, poor patient adherence and a deficient health care system. Additionally, the emergence of multidrug-resistant TB (MDR-TB) and extensively drug-resistant TB (XDR-TB) further erodes the ambitions of the WHO program [1]. In the case of MDR/XDR TB, a wide palette of second- and third-line anti-TB drugs are used, e.g., fluoroquinolones, ethionamide, thioacetazone, clarithromycin and clofazimine [4,5]. However, this 
regimen not only requires more toxic and costly medications; it also requires a longer treatment duration (up to 24 months), resulting in a poor outcome. Moreover, soon after their introduction, resistance has already developed to the newly approved agents bedaquiline [6] and delamanid [7], with the use of pretomanid restricted to a limited and specific population of patients [8]. Thus, novel anti-TB agents are needed to effectively shorten the treatment regime and cure MDR-/XDR-TB.

Thymidylate kinase, a key enzyme for the synthesis of the DNA building block thymidine- $5^{\prime}$ triphosphate, is indispensable for bacterial survival [9-11]. The availability of co-crystal structures of MtbTMPK [12-16] has aided the discovery of MtbTMPK inhibitors, featuring both nucleoside [14,17-20] and non-nucleoside $[14,15,20,21]$ structures.

A previous work from our laboratory started from compound 1 (Figure 1), originally reported by AstraZeneca as an inhibitor of TMPK's of Gram-positive bacteria [22]. After we found that it also potently inhibited $M t b T M P K$, a SAR investigation demonstrated that it could be converted to the achiral inhibitor 2 [14]. Further modifications led to the identification of 1-(1-arylethylpiperidin-4-yl)thymine analog 3, which, compared to $\mathbf{1}$, displayed a nine-fold lower minimum inhibitory concentration (MIC) value for H37Rv [14].

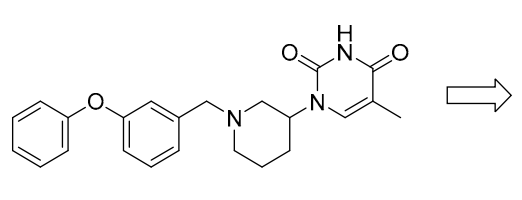

$$
( \pm)-1
$$

$\mathrm{IC}_{50}(\mathrm{~S}$. aureus TMPK) $=156 \mu \mathrm{M}$

$\mathrm{IC}_{50}(\mathrm{~S}$. pneumoniae $\mathrm{TMPK})=3.4 \mu \mathrm{M}$

$\mathrm{IC}_{50}($ MtbTMPK $)=21 \mu \mathrm{M}$

$\mathrm{MIC}(\mathrm{H} 37 \mathrm{Rv})=318.5 \mu \mathrm{M}$

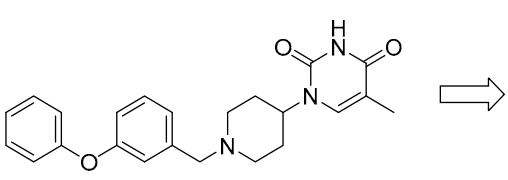

2

$\mathrm{IC}_{50}($ MtbTMPK $)=6.1 \mu \mathrm{M}$<smiles>Cc1cn(C2CCN(CCc3cccc(Oc4ccccc4)c3)CC2)c(=O)[nH]c1=O</smiles>

3

$\mathrm{IC}_{50}($ MtbTMPK $)=11 \mu \mathrm{M}$ $\mathrm{MIC}(\mathrm{H} 37 \mathrm{Rv})=37 \mu \mathrm{M}$

Figure 1. Structures of reported MtbTMPK inhibitors 1, 2 and $\mathbf{3}$ and their TMPK inhibitory potency and antitubercular activity [14,20]. Compound 3 was resynthesized and re-evaluated herein as a reference. MIC: minimum inhibitory concentration.

In this manuscript, we describe our optimization efforts towards finding potent antimycobacterial agents based on 3 by introducing modifications on the A/B/C/D ring (Figure 2) and by altering the linker part. An overview of the synthesized analogs is presented in Figure 2.

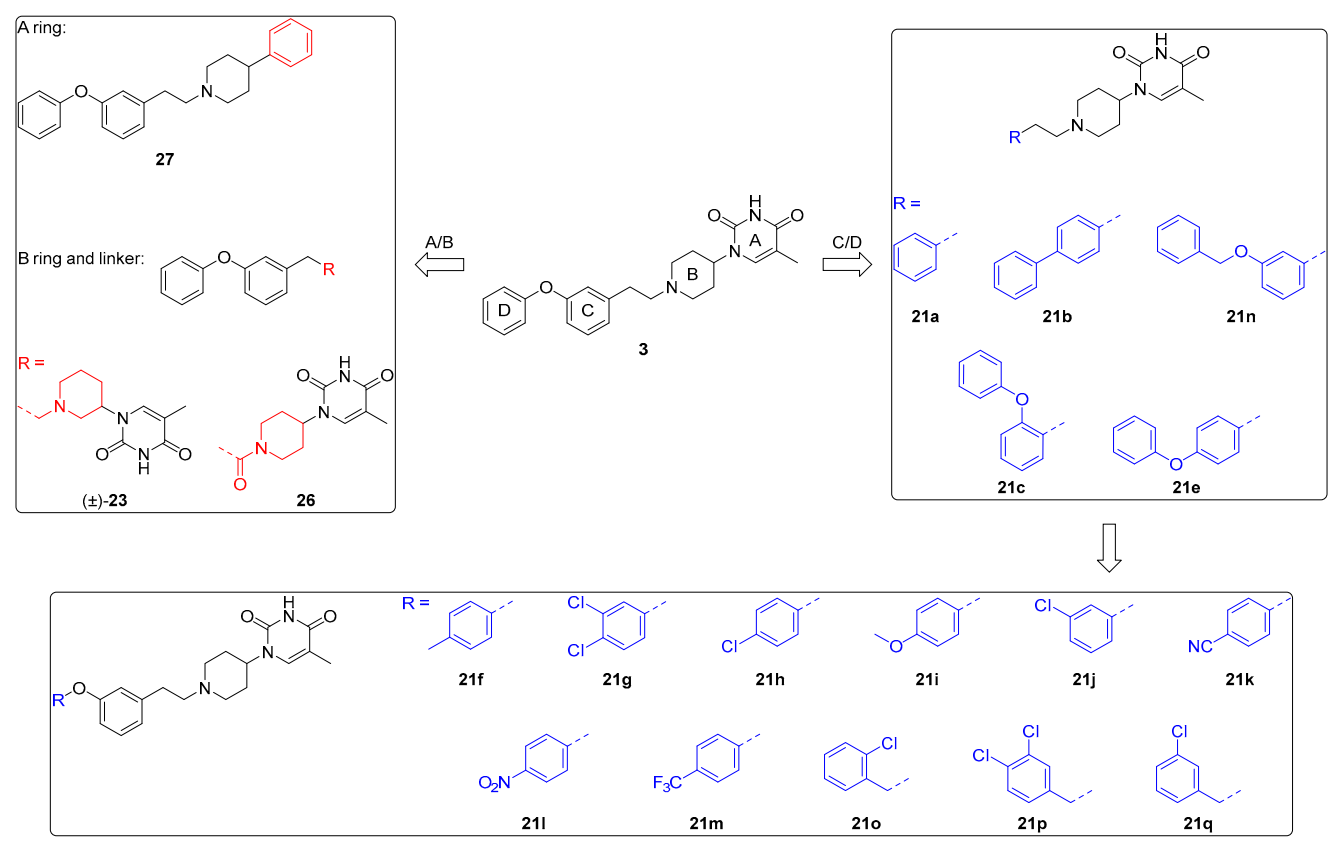

Figure 2. An overview of the synthesized compounds 3, 21a-21q, 26 and 27 in this study. 


\section{Results and Discussion}

\subsection{Chemistry}

The envisioned analogs were synthesized according to a literature-reported procedure [14]. Briefly, the synthesis encompassed the reductive amination of $\mathrm{N}^{3}$-benzyloxymethyl (BOM)-protected piperidinyl thymine with the appropriate aldehyde, followed by trifluoroacetic acid (TFA)-mediated BOM deprotection to yield the desired analogs [14]. The required aldehyde intermediates (Scheme 1) were obtained through the reduction of commercially available substituted methyl phenylacetate esters with $\mathrm{LiAlH}_{4}$ [23] and subsequent re-oxidation by pyridinium chlorochromate (PCC) [24,25]. When the required methyl esters were not commercial available, substituted phenoxy- or benzyloxyaryl analogs were obtained through either Chan-Lam coupling of hydroxyphenylacetic methyl ester with a boronic acid [26] or alkylation of the hydroxyphenylacetic methyl ester with benzyl bromide under basic conditions [27].
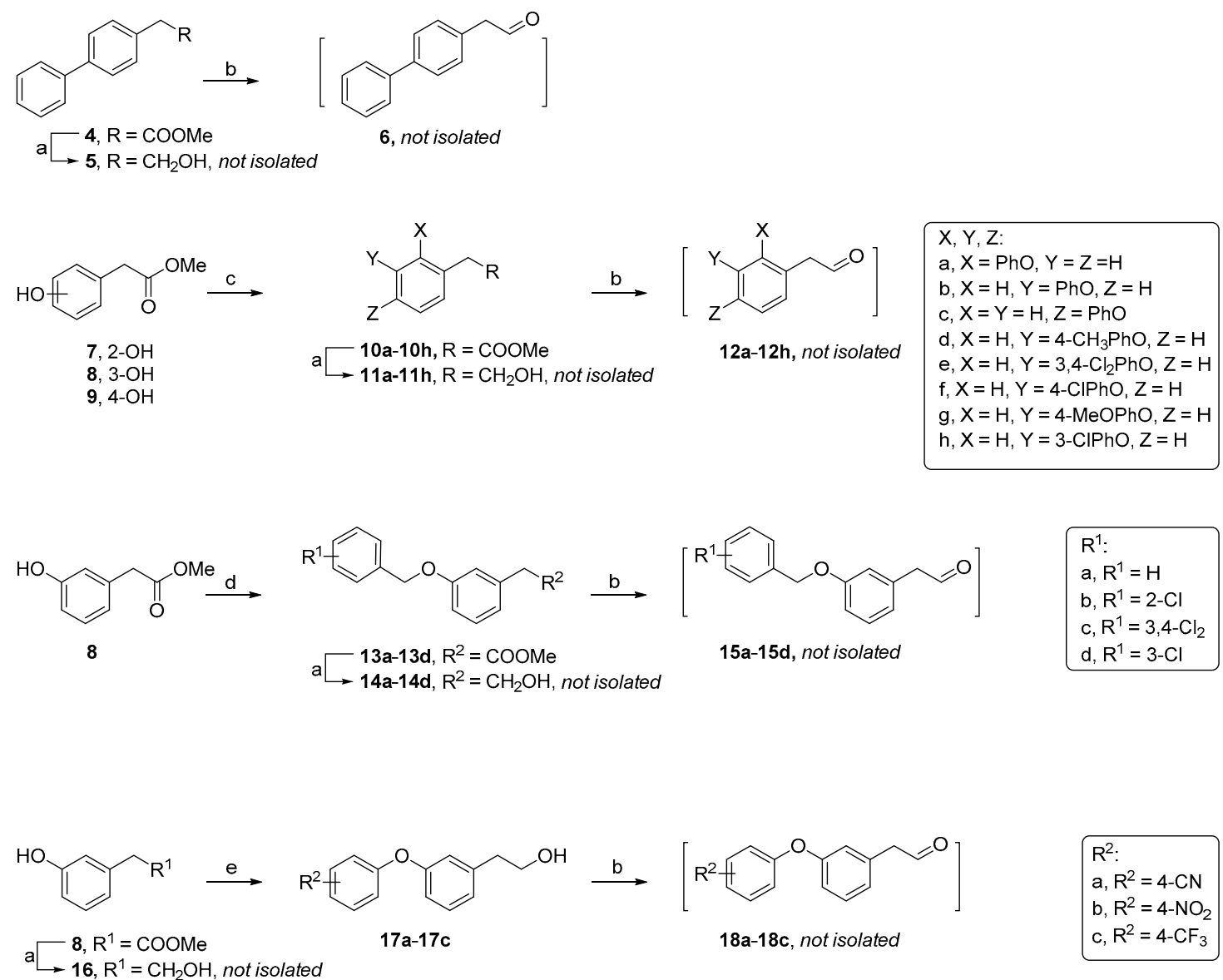

Scheme 1. Synthesis of crude aldehyde intermediates. Reagents and conditions: (a) $\mathrm{LiAlH}_{4}$ and tetrahydrofuran (THF); (b) pyridinium chlorochromate (PCC) and dichloromethane (DCM); (c) substituted phenylboronic acid, pyridine, $4 \AA$ molecular sieves, $\mathrm{Cu}(\mathrm{OAc})_{2}$ and 1,2-dichloroethane; (d) substituted benzyl bromide, $\mathrm{K}_{2} \mathrm{CO}_{3} / \mathrm{Cs}_{2} \mathrm{CO}_{3}, \mathrm{NaI}$ and dimethylformamide (DMF) and (e) substituted fluorobenzene, $\mathrm{K}_{2} \mathrm{CO}_{3}, \mathrm{DMF}$ and $90^{\circ} \mathrm{C}$.

Due to the low yield of 17a-17c in the Chan-Lam coupling reaction, a different synthetic route was applied for the synthesis of aldehydes 18a-18c, which was based on nucleophilic aromatic substitution of the phenol with the appropriate 4-subsituted fluorobenzene [28]. Then, a PCC-mediated oxidation furnished the desired aldehydes, which were used without further purification in the reductive amination step (Scheme 2). 


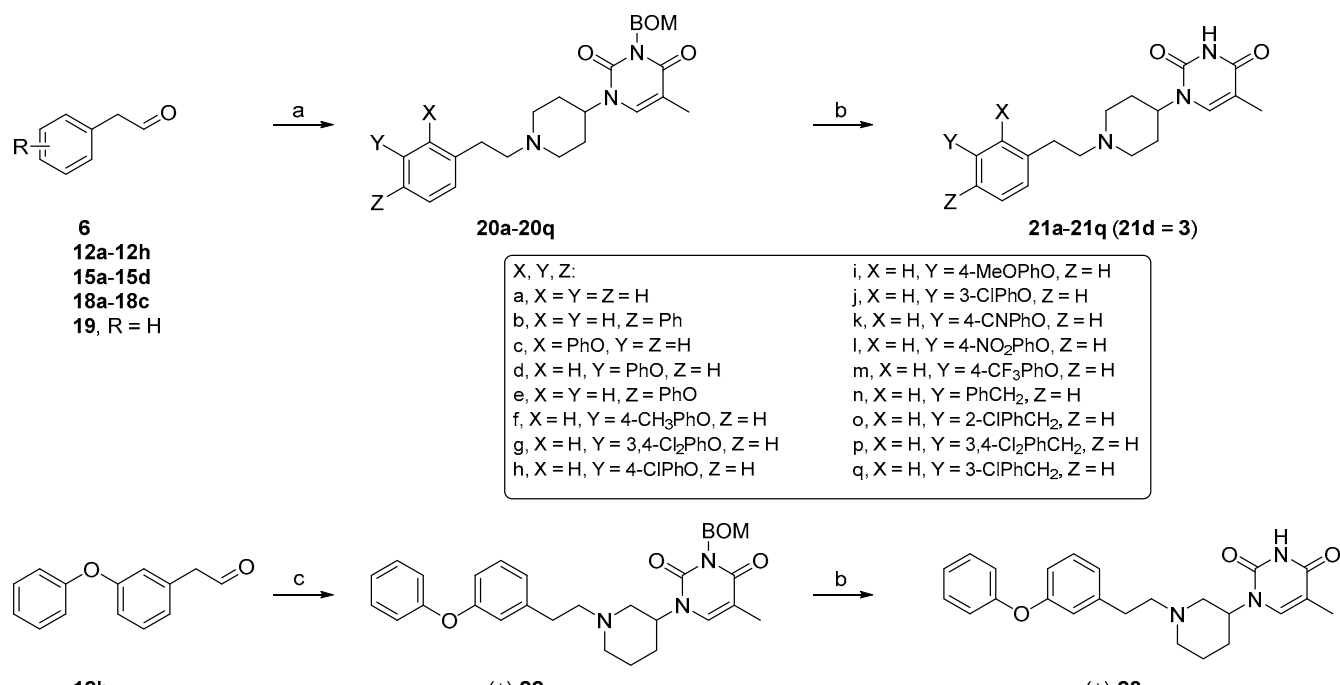

$12 \mathrm{~b}$
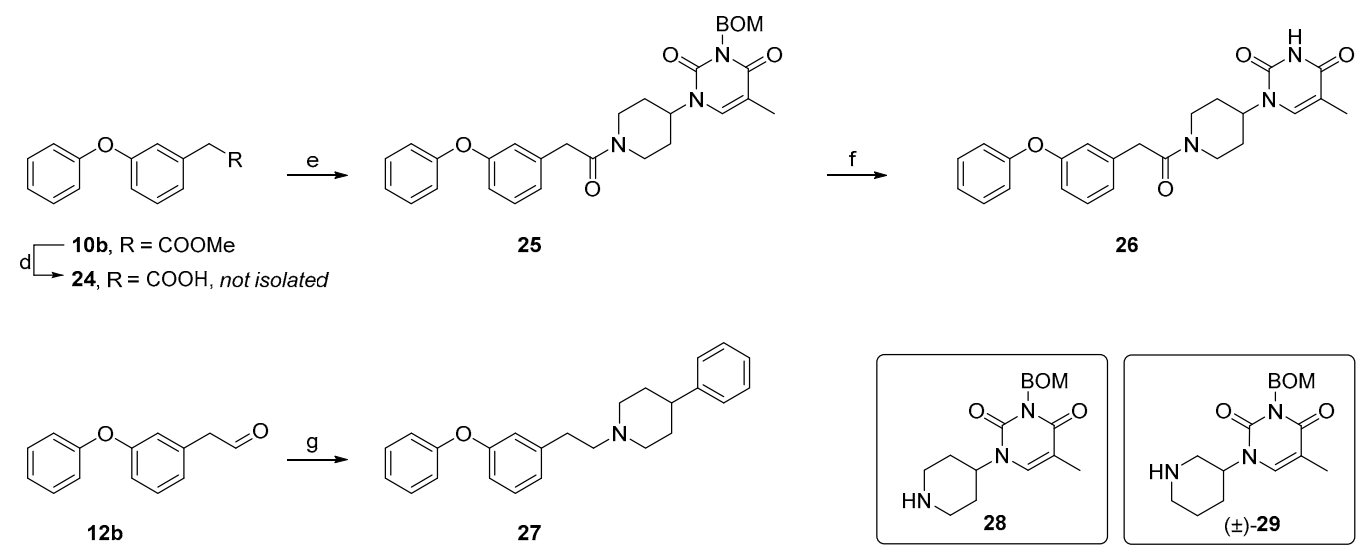

Scheme 2. Synthesis of the final compounds. Reagents and conditions: (a) 28, sodium triacetoxyborohydride and 1,2-dichloroethane; (b) trifluoroacetic acid (TFA), $\mathrm{Et}_{3} \mathrm{SiH}$ and $73{ }^{\circ} \mathrm{C}$; (c) ( \pm )-29, sodium triacetoxyborohydride and 1,2-dichloroethane; (d) (i) aq. $\mathrm{NaOH}, \mathrm{MeOH}$ and $50{ }^{\circ} \mathrm{C}$; (ii) $1 \mathrm{~N}$ aq. $\mathrm{HCl}$ and $\mathrm{H}_{2} \mathrm{O}$; (e) 28, N-(3-dimethylaminopropyl)- $\mathrm{N}^{\prime}$-ethylcarbodiimide hydrochloride (EDC. $\mathrm{HCl}$ ), 4-dimethylaminopyridine (DMAP) and DCM; (f) Pd/C (10\%), $\mathrm{H}_{2}, \mathrm{HCOOH}(0.5 \%)$ and isopropanol/ $\mathrm{H}_{2} \mathrm{O}(10 / 1 \mathrm{v} / \mathrm{v})$ and $(\mathrm{g})$ 4-phenylpiperidine, sodium triacetoxyborohydride and 1,2-dichloroethane.

Compounds 21a-21q and 23 were synthesized via reductive amination of $\mathrm{N}^{3}$-BOM-protected piperidinyl thymine with the appropriate aldehyde [14], followed by trifluoroacetic acid (TFA)-mediated BOM deprotection (Scheme 2) [29]. Potential dimerization during the TFA-mediated deprotection [14] was precluded by adding $\mathrm{Et}_{3} \mathrm{SiH}$ as a cation scavenger [29].

Amide 26 was obtained via N-(3-dimethylaminopropyl)- $\mathrm{N}^{\prime}$-ethylcarbodiimide (EDC)-mediated coupling of 28 with 2-(3-phenoxyphenyl)acetic acid, which was obtained by hydrolysis of the corresponding methyl ester. The BOM group in the resulting amide intermediate was removed by catalytic hydrogenation with $\mathrm{Pd} / \mathrm{C}$ [30].

A one-step reductive amination of 4-phenylpiperidine with aldehyde $\mathbf{1 2} \mathbf{b}$ afforded the phenyl analog 27.

\subsection{Biological Activity}

All synthesized compounds were evaluated for their capacity to inhibit MtbTMPK. Our previously reported analog 3 , the starting point, was resynthesized and its reported inhibitory potency confirmed 
(Table $1, \mathrm{IC}_{50}$ values of 11 and $17 \mu \mathrm{M}$, respectively) [14]. Replacement of the thymine ring by a phenyl (27) led to a complete loss of the inhibitory potency. Additionally, repositioning of the thymine ring from the para to the meta-position of the piperidine ring [20,22] (racemic compound 23) resulted in a substantial (>10-fold) loss in the inhibitory potency. In-line with earlier observations, with a one-carbon linker [14,21], amide analog 26 displayed a weak enzyme inhibition.

Table 1. Mycobacterium tuberculosis TMPK (MtbTMPK) inhibitory potency of the compounds.

Compound

${ }^{a}$ Values for which standard deviations are given are the calculated mean values of at least two measurements. ${ }^{\mathrm{b}}$ NI: no inhibition at a concentration of $0.2 \mathrm{mM}$.

Having established that 1-(1-arylethylpiperidin-4-yl)thymine is preferable for inhibitory potency, our efforts were then directed toward the exploration of the biphenyl ether tail. Deletion of the terminal phenoxy group (analog 21a) resulted in a 40-fold decrease in inhibitory potency. Docking studies based on the X-ray co-crystal structure of MtbTMPK with our previously reported 1-(piperidin-4-yl)thymine inhibitor (PDB 5NR7) [14] were performed to rationalize the observed SAR. Docking indicated that the loss of a hydrophobic interaction with Tyr39 accounts for the drop in inhibitory potency (Figure 3A). The addition of a methylene moiety between the terminal phenyl ring and C-phenoxy group (analog 21n) caused a five-fold decrease in the inhibitory potency. The docking pose of compound 21n in $M t b T M P K$ (Figure 3B) showed that the elongated benzyloxy phenyl resulted in a weaker hydrophobic interaction with Tyr39 than the phenoxy moiety of 3 . Omitting the oxygen atom between the two phenyl rings led to a complete loss of enzyme inhibitory potency (compound 21b). Moreover, moving the terminal phenoxy ring of 3 to the ortho or para-position of ring $C$ (compounds $\mathbf{2 1 c} / \mathbf{2 1 e}$ ) had a negative effect on the inhibitory activity. 
A

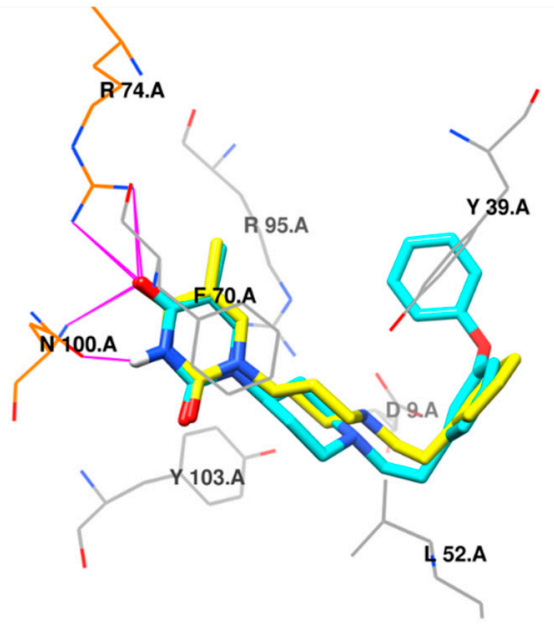

B

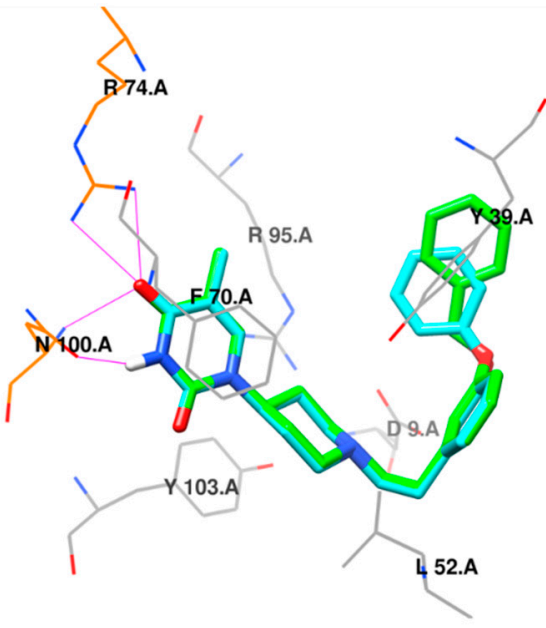

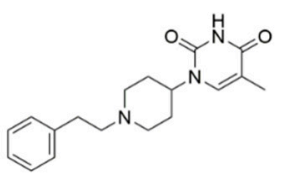

21a

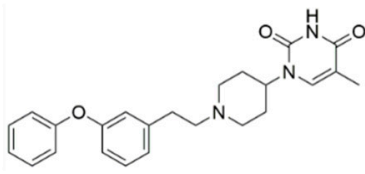

3

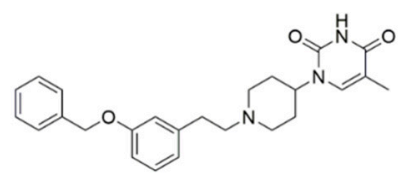

$21 n$

Figure 3. (A) Structure overlay of compound 3 (cyan) and 21a (yellow) docked in the MtbTMPK (PDB 5NR7) [14] active site. (B) Structure overlay of compound 3 (cyan) and 21n (green) docked in the MtbTMPK (PDB 5NR7) [14] active site. All residues interacting with the inhibitors, including the hydrophobic contact (gray wire) and hydrogen-bonding interaction (residues in orange wire, hydrogen bonds indicated in magenta), were calculated using LigPlus [31]. Illustration was created using Chimera [32].

Based on these results, further optimization efforts focused on the substitution pattern of the terminal phenyl ring of $\mathbf{3}$ and $\mathbf{2 1}$. As shown in Table 2, most of the substituted analogs exhibited a small decrease in inhibitory activity compared to 3 . Of note, the introduction of sterically demanding electron withdrawing substituents $(\mathbf{2 1 k} / \mathbf{2 1 1} / \mathbf{2 1} \mathrm{m})$ resulted in a significant drop in $M t b T M P K$ inhibitory potency.

Table 2. MtbTMPK inhibitory activity of the compounds.

Compound

a Values for which standard deviations are given are the calculated mean values of at least two measurements. b Solubility issue. 
Interestingly, the introduction of a 3-chloro (21j) but not a 4-chloro substituent (21h) afforded a significant improvement in the inhibitory potency due to an edge-to-face $\pi$-stacking interaction between the 3-chlorobenzene ring and Tyr39, as found for compound 3 (Figure 4). Introduction of a 3-chloro substituent in the benzyl analog $21 n$ also had a beneficial impact on the inhibitory activity.
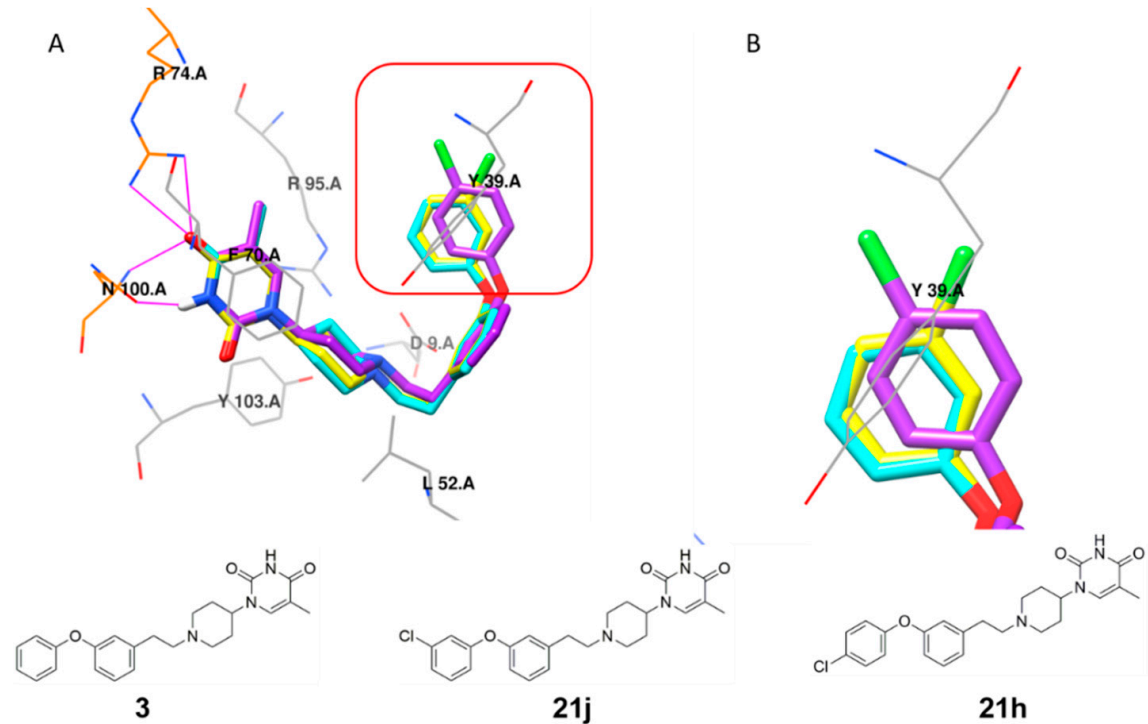

Figure 4. (A) Structure overlay of compound 3 (cyan), 21j (yellow) and $\mathbf{2 1 h}$ (purple) in the MtbTMPK (PDB 5NR7) [14] active site. (B) Detailed representation of the interactions of the D rings of 3, 21h and 21j, with the side chain of Tyr39. All residues interacting with the inhibitors, including the hydrophobic contact (gray wire) and hydrogen-bonding interaction (residues in orange wire, hydrogen bonds indicated in magenta), were calculated using LigPlus [31]. Illustration was created using Chimera [32].

Finally, all compounds were evaluated for their in vitro antimycobacterial activity (Table 3). Consistent with the observations on the enzyme inhibitory activities, modifications of the scaffold of 3 did not yield analogs with superior antimycobacterial activity (21a-21c, 21e, 21n, 23 and 26). Remarkably, analog 27 , in which the thymine moiety is replaced by a phenyl ring, showed potent antimycobacterial activity but lacked selectivity, as evidenced by its equipotent cytotoxicity. The introduction of substituents on the distal phenyl ring of 3 afforded several analogs with improved antimycobacterial activity (21f-21j). Substitution of the D-ring of the benzyloxy analog $\mathbf{2 1 n}$ also contributed to the growth inhibitory activity. However, the selectivity vs. MRC-5 fibroblasts was modest.

Table 3. Antimycobacterial activity against H37Rv and cytotoxicity against MRC-5 fibroblasts of the compounds in this study.

\begin{tabular}{|c|c|c|c|c|c|c|c|}
\hline Compound & $\begin{array}{c}\text { MIC }^{a} \\
(\mathrm{H} 37 \mathrm{Rv}, \mu \mathrm{M})\end{array}$ & $\begin{array}{c}\mathrm{IC}_{50} \\
(\mathrm{MRC}-5, \mu \mathrm{M})\end{array}$ & $S_{I^{b}}^{b}$ & Compound & $\begin{array}{c}\text { MIC } \\
(\mathrm{H} 37 \mathrm{Rv}, \mu \mathrm{M})\end{array}$ & $\begin{array}{c}\mathrm{IC}_{50} \\
(\mathrm{MRC}-5, \mu \mathrm{M})\end{array}$ & SI \\
\hline 3 & 37 & $>64$ & $>1.73$ & $21 k$ & $\geq 50$ & $>64$ & - \\
\hline $21 a$ & $>50$ & $>64$ & - & 211 & 37 & $>64$ & $>1.73$ \\
\hline $21 b$ & $>50$ & 22.22 & - & $21 \mathrm{~m}$ & 37 & $>64$ & $>1.73$ \\
\hline $21 c$ & 50 & 26.81 & 0.54 & $21 n$ & 37 & 27.57 & 0.74 \\
\hline $21 \mathrm{e}$ & $>50$ & $>64$ & - & 210 & 19 & 7.08 & 0.37 \\
\hline $21 f$ & 19 & 8.42 & 0.44 & $21 p$ & 12.5 & 4.22 & 0.34 \\
\hline $21 \mathrm{~g}$ & 19 & 5.66 & 0.30 & $21 q$ & 25 & 19.84 & 0.79 \\
\hline $21 \mathrm{~h}$ & 12.5 & 5.81 & 0.46 & 23 & $>50$ & 21.77 & - \\
\hline $21 \mathrm{i}$ & 25 & $>64$ & $>2.56$ & 26 & $>50$ & $>64$ & - \\
\hline $21 j$ & 19 & 25.24 & 1.33 & 27 & 6.25 & 8.06 & 1.29 \\
\hline
\end{tabular}

${ }^{a}$ Minimum inhibitory concentration (MIC) is the minimum concentration required to inhibit $>99 \%$ growth of Mycobacterium tuberculosis H37Rv in the liquid culture. ${ }^{\mathrm{b}}$ Selectivity index (SI): $\mathrm{IC}_{50}$ (MRC-5)/MIC (H37Rv). 


\section{Materials and Methods}

\subsection{Enzymatic Assay}

The enzymatic assay was performed as previously reported on the recombinant purified MtbTMPK [14,33]. As described by Blondin et al. [34], compounds were evaluated by a serial dilution method using the spectrophotometric assay at fixed concentrations of adenosine triphosphate (ATP) $(0.5 \mathrm{mM})$ and thymidine monophosphate (dTMP; $0.05 \mathrm{mM})$. The reaction medium includes 50-mM Tris- $\mathrm{HCl} ; \mathrm{pH} 7.4 ; 50-\mathrm{mM} \mathrm{KCl} ; 2-\mathrm{mM} \mathrm{MgCl}_{2} ; 0.2-\mathrm{mM}$ nicotinamide adenine dinucleotide (NADH); 1-mM phosphoenol pyruvate and 2 units each of coupling enzymes (lactate dehydrogenase, pyruvate kinase and nucleoside diphosphate kinase). From the experimental data, the $\mathrm{IC}_{50}$ value was calculated using KaleidaGraph 4.5.3.

\subsection{Computational Studies}

For the molecular modeling, X-ray structure of the MtbTMPK (PDB entry 5NR7 [14]) was analyzed using AutoDock vina and AutodockTools-1.5.6. [35]. In ChemDraw 3D 16.0, the PDB files of all ligands were generated after the energy was minimized (minimum RMS gradient: 0.001). The PDBQT file of the ligands and receptors were prepared by AutodockTools-1.5.6, including atom types, atomic partial charges and the information on the ligand torsional degrees. Using a grid spacing of 0.375 and $60 \times 60 \times 60$ numbers of grid points, the prepared PDBQT files of ligands and receptors were docked (centered on the MtbTMPK active site PHE70 CE2, the coordinates $\mathrm{x}, \mathrm{y}$ and $\mathrm{z}$ were $-0.997,26.240$ and -4.528, correspondingly) through the Lamarckian 4.2 method. Each ligand was docked in Autodock vina 3 times, with each time generating 20 possible conformations. Chimera in combination with LigPlus were used to analyze the results.

\subsection{In Vitro Antituberculosis Assay}

The MIC values of all compounds were determined as previously described [36]. In brief, M. tuberculosis H37Rv (ATCC 27294) was grown to optical density at $650 \mathrm{~nm}$ wavelength $\left(\mathrm{OD}_{650 \mathrm{~nm}}\right)$ 0.2 in Middlebrook $7 \mathrm{H} 9$ medium supplemented with $0.4 \%$ glucose, $0.03 \%$ Bacto casitone and $0.05 \%$ Tyloxapol (7H9/glucose/casitone/Tyloxapol) prior to further 1000-fold dilution in fresh medium. Drugs were 2-fold serially diluted in duplicate in 7H9/glucose/casitone/Tyloxapol (50 $\mu \mathrm{L} /$ well) in a concentration range spanning 100-0.049 $\mu \mathrm{M}$ in sterile 96-well U-bottom clear polystyrene microtiter plates. Isoniazid and DMSO as positive and negative controls, respectively. An equal volume $(50 \mu \mathrm{L})$ of diluted cells was added to the plates with the serial drug dilution. Plates were sealed in Ziplock bags and incubated at $37^{\circ} \mathrm{C}$. After 7-14 days, plates were read with an enlarging inverted mirror plate reader. The MIC was recorded as the concentration that fully inhibited all visible growth.

\subsection{In Vitro Cytotoxicity Assay}

The cytotoxicity of compounds on MRC-5 fibroblasts was performed exactly as previously reported [14].

\subsection{Chemistry}

All reagents and solvents were purchased from standard commercial sources and were of analytical grade. All synthetic compounds described in this study were checked with analytical TLC (Macherey-Nagel precoated F254 aluminum plates, Düren, Germany), visualized under UV light at $254 \mathrm{~nm}$ and purified by column chromatography (CC) on a Reveleris X2 (Grace, BÜCHI, Flawil, Switzerland) automated flash unit. All final compounds and some intermediates were measured with Varian Mercury 300/75 MHz (Palo Alto, CA, USA) or a Bruker AVANCE (Fällanden, Zürich, Switzerland) $\mathrm{Neo}^{\circledR}$ 400/100 MHz spectrometer at 298.15 K using tetramethylsilane (TMS) as an internal standard. The analysis and confirmation of the final compounds were conducted with ${ }^{1} \mathrm{H},{ }^{13} \mathrm{C}$, 
HSQC and HMBC NMR spectral data (Supplementary Materials). High-resolution mass spectrometry was performed on a Waters LCT Premier XE ${ }^{\mathrm{TM}}$ (Waters, Zellik, Belgium) time-of-flight (TOF) mass spectrometer equipped with a standard electrospray ionization (ESI) and modular LockSpray ${ }^{\mathrm{TM}}$ interface (Waters, Zellik, Belgium). The purity of the tested compounds was determined by LC-MS analysis using a Waters AutoPurification system equipped with a Waters Cortecs C18 column $(2.7 \mu \mathrm{m}$, $100 \times 4.6 \mathrm{~mm})$, as was a gradient system of formic acid in $\mathrm{H}_{2} \mathrm{O}(0.2 \%, v / v) / \mathrm{MeCN}$ with a gradient of 95:5 to $0: 100$ in $6.5 \mathrm{~min}$ at a flow rate of $1.44 \mathrm{~mL} / \mathrm{min}$.

General procedure A: Synthesis of biphenyl ether aldehyde building blocks. According to a literature report [26], hydroxyphenylacetic ester derivatives (1.0 eq), phenylboronic acid derivatives (3.0 eq.), $\mathrm{Cu}(\mathrm{OAc})_{2}$ (2.0 eq.), $4 \AA$ molecular sieves $(0.18 \mathrm{~g} / \mathrm{mmol}$ ester $)$ and pyridine (3.0 eq.) in 1,2-dichloroethane $(6.0 \mathrm{~mL} / \mathrm{mmol}$ ester $)$ afforded the biphenyletheracetic ester intermediates. To a solution of the biphenyletheracetic ester intermediates (1.0 eq.) in dry tetrahydrofuran (THF) $\left(6.0 \mathrm{~mL} / \mathrm{mmol}\right.$ ester intermediate) was added $\mathrm{LiAlH}_{4}\left(2.0\right.$ eq.) at $0{ }^{\circ} \mathrm{C}$ under $\mathrm{N}_{2}$ atmosphere, and the resulting mixture was then stirred at room temperature for $1 \mathrm{~h}$ [23]. After complete consumption of the starting material, the reaction mixture was quenched with aq. $\mathrm{Na}^{+} / \mathrm{K}^{+}$tartrate solution $(5.0 \mathrm{~mL} / \mathrm{mmol}$ $\mathrm{LiAlH}_{4}$ ), and the mixture was stirred at room temperature overnight and then filtered. The collected filtrate was dried and concentrated to afford a crude alcohol intermediate, which was oxidized by PCC (2.0 eq.) for $2 \mathrm{~h}$ in dichloromethane (DCM) $(5.0 \mathrm{~mL} / \mathrm{mmol}$ PCC) [24,25]. The reaction mixture was filtered through a short silica column. The collected filtrate was concentrated in vacuo and used in the next step without any additional purification.

General procedure B: Synthesis of final compounds. To a solution of aldehyde intermediates (1.0 eq.) and 28 (1.0 eq.) in 1,2-dichloroethane (33 mL/mmol aldehyde) was added sodium triacetoxyborohydride (2.0 eq.). The resulting mixture was stirred at room temperature for overnight to afford the BOM-protected intermediate [14], which was deprotected with TFA (17 mL/mmol aldehyde) in the presence of triethylsilane [29] $(17 \mathrm{~mL} / \mathrm{mmol}$ aldehyde $)$ at $73{ }^{\circ} \mathrm{C}$ for $4 \mathrm{~h}$. After cooling down to room temperature, the reaction mixture was evaporated in vacuo to remove TFA, and the residue was adjusted to $\mathrm{pH} 6$ with $1 \mathrm{~N}$ aq. $\mathrm{HCl}$. Purification of the resulting mixture by column chromatography gave the desired compounds.

2-([1,1'-Biphenyl]-4-yl)acetaldehyde (6). To a solution of methyl 2-([1,1'-biphenyl]-4-yl)acetate (0.3 g, $1.3 \mathrm{mmol})$ in dry THF $(7.8 \mathrm{~mL})$ was added $\mathrm{LiAlH}_{4}(0.10 \mathrm{~g}, 2.7 \mathrm{mmol})$ to give alcohol intermediate, which was oxidized with PCC $(0.56 \mathrm{~g}, 2.6 \mathrm{mmol})$ in DCM $(13.0 \mathrm{~mL})$ to yield aldehyde $6\left(\mathrm{C}_{14} \mathrm{H}_{12} \mathrm{O}\right.$, $0.22 \mathrm{~g}, 1.1 \mathrm{mmol})$.

2-(2-Phenoxyphenyl)acetaldehyde (12a). Following the general procedure A, methyl 2-(2-hydroxyphenyl)acetate $(1.1 \mathrm{~g}, 8.1 \mathrm{mmol})$, phenylboronic acid $(2.9 \mathrm{~g}, 24 \mathrm{mmol}), \mathrm{Cu}(\mathrm{OAc})_{2}$ (2.9 g, $16 \mathrm{mmol}), 4 \AA$ molecular sieves $(1.5 \mathrm{~g})$ and pyridine $(1.9 \mathrm{~mL}, 24 \mathrm{mmol})$ in 1,2-dichloroethane $(49 \mathrm{~mL})$ afforded the ester intermediate methyl 2-(2-phenoxyphenyl)acetate 10a (eluent system: 10\% ethylacetate in petroleum ether, $\mathrm{C}_{15} \mathrm{H}_{14} \mathrm{O}_{3}, 0.40 \mathrm{~g}, 1.6 \mathrm{mmol}, 21 \%$ yield). ${ }^{1} \mathrm{H} \mathrm{NMR}\left(300 \mathrm{MHz}, \mathrm{CDCl}_{3}\right)$ $\delta$ ppm $3.63\left(\mathrm{~s}, 3 \mathrm{H}, \mathrm{OCH}_{3}\right), 3.72\left(\mathrm{~s}, 2 \mathrm{H}, \mathrm{CH}_{2}\right), 6.90(\mathrm{dd}, J=8.1,1.0 \mathrm{~Hz}, 1 \mathrm{H}, \mathrm{Ph}), 6.95-7.01(\mathrm{~m}, 2 \mathrm{H}$, $\mathrm{Ph}), 7.06-7.15$ (m, $2 \mathrm{H}, \mathrm{Ph}), 7.21-7.37$ (m, $4 \mathrm{H}, \mathrm{Ph}) .{ }^{13} \mathrm{C} \mathrm{NMR}\left(75 \mathrm{MHz}, \mathrm{CDCl}_{3}\right) \delta \mathrm{ppm} 35.6\left(1 \mathrm{C}, \mathrm{CH}_{2}\right)$, $51.8\left(1 \mathrm{C}_{,} \mathrm{OCH}_{3}\right), 118.3$ (2 C, Ph), 118.8 (1 C, Ph), 123.0 (1 C, Ph), 123.6 (1 C, Ph), 125.8 (1 C, Ph), 128.6 (1 C, Ph), 129.6 (2 C, Ph), 131.4 (1 C, Ph), 155.0 (1 C, Ph), 157.2 (1 C, Ph), 171.7 (1 C, CO). Then, 10a $(0.20 \mathrm{~g}, 0.83 \mathrm{mmol})$ was treated with $\mathrm{LiAlH}_{4}(63 \mathrm{mg}, 1.7 \mathrm{mmol})$ in dry THF $(5.0 \mathrm{~mL})$ to give alcohol intermediate, which was oxidized with PCC $(0.34 \mathrm{~g}, 1.6 \mathrm{mmol})$ in DCM $(8.0 \mathrm{~mL})$ to yield aldehyde 12a $\left(\mathrm{C}_{14} \mathrm{H}_{12} \mathrm{O}_{2}, 0.15 \mathrm{~g}, 0.70 \mathrm{mmol}\right)$.

2-(3-Phenoxyphenyl)acetaldehyde (12b). Following the general procedure A, methyl 2-(3-hydroxyphenyl)acetate $(1.1 \mathrm{~g}, 8.1 \mathrm{mmol})$, phenylboronic acid $(2.9 \mathrm{~g}, 24 \mathrm{mmol}), \mathrm{Cu}(\mathrm{OAc})_{2}$ (2.9 g, $16 \mathrm{mmol}), 4 \AA$ molecular sieves $(1.5 \mathrm{~g})$ and pyridine $(1.9 \mathrm{~mL}, 24 \mathrm{mmol})$ in 1,2-dichloroethane $(49 \mathrm{~mL})$ afforded the ester intermediate methyl 2-(3-phenoxyphenyl)acetate 10b (eluent system: 10\% ethylacetate in petroleum ether, $\mathrm{C}_{15} \mathrm{H}_{14} \mathrm{O}_{3}, 0.80 \mathrm{~g}, 3.3 \mathrm{mmol}, 41 \%$ yield $) .{ }^{1} \mathrm{H} \mathrm{NMR}\left(300 \mathrm{MHz}, \mathrm{CDCl}_{3}\right)$ 
Sppm $3.62\left(\mathrm{~s}, 2 \mathrm{H}, \mathrm{CH}_{2}\right), 3.71\left(\mathrm{~s}, 3 \mathrm{H}, \mathrm{OCH}_{3}\right), 6.90-6.95(\mathrm{~m}, 1 \mathrm{H}, \mathrm{Ph}), 6.98(\mathrm{t}, J=2.1 \mathrm{~Hz}, 1 \mathrm{H}, \mathrm{Ph})$, 7.01-7.07 (m, $3 \mathrm{H}, \mathrm{Ph}), 7.13(\mathrm{tt}, J=7.4,1.1 \mathrm{~Hz}, 1 \mathrm{H}, \mathrm{Ph}), 7.26-7.40(\mathrm{~m}, 3 \mathrm{H}, \mathrm{Ph}) .{ }^{13} \mathrm{C}$ NMR $(75 \mathrm{MHz}$, $\left.\mathrm{CDCl}_{3}\right)$ Sppm 40.9 (1 C, $\left.\mathrm{CH}_{2}\right), 52.0\left(1 \mathrm{C}, \mathrm{OCH}_{3}\right), 117.3$ (1 C, Ph), 119.0 (2 C, Ph), 119.7 (1 C, Ph), 123.3 (1 C, Ph), 124.0 (1 C, Ph), 129.7 (3 C, Ph), 135.7 (1 C, Ph), 156.9 (1 C, Ph), 157.4 (1 C, Ph), 171.6 (1 C, $\mathrm{CO})$. Then, $10 \mathrm{~b}(0.20 \mathrm{~g}, 0.83 \mathrm{mmol})$ was treated with $\mathrm{LiAlH}_{4}(63 \mathrm{mg}, 1.7 \mathrm{mmol})$ in dry THF $(5.0 \mathrm{~mL})$ to give alcohol intermediate, which was oxidized with PCC $(0.35 \mathrm{~g}, 1.6 \mathrm{mmol})$ in DCM $(8.0 \mathrm{~mL})$ to yield aldehyde $\mathbf{1 2 b}\left(\mathrm{C}_{14} \mathrm{H}_{12} \mathrm{O}_{2}, 0.15 \mathrm{~g}\right.$, $\left.0.71 \mathrm{mmol}\right)$.

2-(4-Phenoxyphenyl)acetaldehyde (12c). Following the general procedure A, methyl 2-(4-hydroxyphenyl)acetate $(0.60 \mathrm{~g}, 4.4 \mathrm{mmol})$, phenylboronic acid $(1.6 \mathrm{~g}, 13 \mathrm{mmol}), \mathrm{Cu}(\mathrm{OAc})_{2}$ $(1.6 \mathrm{~g}, 8.8 \mathrm{mmol}), 4 \AA$ molecular sieves $(0.70 \mathrm{~g})$ and pyridine $(1.1 \mathrm{~mL}, 13 \mathrm{mmol})$ in 1,2-dichloroethane $(26 \mathrm{~mL})$ afforded the ester intermediate methyl 2-(4-phenoxyphenyl)acetate 10c (eluent system: 10\% ethylacetate in petroleum ether, $\mathrm{C}_{15} \mathrm{H}_{14} \mathrm{O}_{3}, 0.66 \mathrm{~g}, 2.7 \mathrm{mmol}, 62 \%$ yield). ${ }^{1} \mathrm{H}$ NMR ( $\left.300 \mathrm{MHz}, \mathrm{CDCl}_{3}\right) \delta$ ppm 3.63 (s, $\left.2 \mathrm{H}, \mathrm{CH}_{2}\right), 3.72\left(\mathrm{~s}, 3 \mathrm{H}, \mathrm{OCH}_{3}\right), 6.95-7.06(\mathrm{~m}, 4 \mathrm{H}, \mathrm{Ph}), 7.08-7.15(\mathrm{~m}, 1 \mathrm{H}, \mathrm{Ph}), 7.23-7.29(\mathrm{~m}$, $2 \mathrm{H}, \mathrm{Ph}), 7.31-7.39(\mathrm{~m}, 2 \mathrm{H}, \mathrm{Ph}) .{ }^{13} \mathrm{C}$ NMR $\left(75 \mathrm{MHz}, \mathrm{CDCl}_{3}\right) \delta \mathrm{ppm} 40.3\left(1 \mathrm{C} \mathrm{CH}_{2}\right), 52.0\left(1 \mathrm{C}_{,} \mathrm{OCH}_{3}\right)$, 118.8 (4 C, Ph), 123.2 (1 C, Ph), 128.7 (1 C, Ph), 129.6 (2 C, Ph), 130.5 (2 C, Ph), 156.3 (1 C, Ph), 157.0 (1 C, $\mathrm{Ph}), 172.0(1 \mathrm{C}, \mathrm{CO})$. Then, $10 \mathrm{c}(0.20 \mathrm{~g}, 0.83 \mathrm{mmol})$ was treated with $\mathrm{LiAlH}_{4}(63 \mathrm{mg}, 1.6 \mathrm{mmol})$ in dry THF (5.0 mL) to give alcohol intermediate, which was oxidized with PCC $(0.3 \mathrm{~g}, 1.4 \mathrm{mmol})$ in DCM (7.0 mL) to yield aldehyde $12 \mathrm{c}\left(\mathrm{C}_{14} \mathrm{H}_{12} \mathrm{O}_{2}, 0.13 \mathrm{~g}, 0.62 \mathrm{mmol}\right)$.

2-(3-(p-Tolyloxy)phenyl)acetaldehyde (12d). Following the general procedure A, methyl 2-(3-hydroxyphenyl)acetate $(0.60 \mathrm{~g}, 4.4 \mathrm{mmol}), p$-tolylboronic acid $(1.8 \mathrm{~g}, 13 \mathrm{mmol}), \mathrm{Cu}(\mathrm{OAc})_{2}(1.6 \mathrm{~g}$, $8.8 \mathrm{mmol}), 4 \AA$ molecular sieves $(0.79 \mathrm{~g})$ and pyridine $(1.1 \mathrm{~mL}, 13 \mathrm{mmol})$ in 1,2-dichloroethane $(26 \mathrm{~mL})$ afforded the ester intermediate methyl 2-(3-(p-tolyloxy)phenyl)acetate 10d (eluent system: 10\% ethylacetate in petroleum ether, $\mathrm{C}_{16} \mathrm{H}_{16} \mathrm{O}_{3}, 0.34 \mathrm{~g}, 1.3 \mathrm{mmol}, 30 \%$ yield). ${ }^{1} \mathrm{H}$ NMR $\left(300 \mathrm{MHz}, \mathrm{CDCl}_{3}\right) \delta$ ppm $2.37\left(\mathrm{~s}, 3 \mathrm{H}, \mathrm{CH}_{3}\right), 3.62\left(\mathrm{~s}, 2 \mathrm{H}, \mathrm{CH}_{2}\right), 3.72\left(\mathrm{~s}, 3 \mathrm{H}, \mathrm{OCH}_{3}\right), 6.89-6.99(\mathrm{~m}, 4 \mathrm{H}, \mathrm{Ph}), 7.00-7.05(\mathrm{~m}, 1 \mathrm{H}$, $\mathrm{Ph}), 7.14-7.21(\mathrm{~m}, 2 \mathrm{H}, \mathrm{Ph}), 7.25-7.32(\mathrm{~m}, 1 \mathrm{H}, \mathrm{Ph}) .{ }^{13} \mathrm{C} \mathrm{NMR}\left(75 \mathrm{MHz}, \mathrm{CDCl}_{3}\right) \delta \mathrm{ppm} 20.6\left(1 \mathrm{C} \mathrm{CH}_{3}\right)$, $40.9\left(1 \mathrm{C}, \mathrm{CH}_{2}\right), 51.9\left(1 \mathrm{C}, \mathrm{OCH}_{3}\right), 116.7$ (1 C, Ph), 119.1 (3 C, Ph), 123.5 (1 C, Ph), $129.6(1 \mathrm{C}, \mathrm{Ph}), 130.1$ (2 C, Ph), 132.9 (1 C, Ph), 135.6 (1 C, Ph), 154.4 (1 C, Ph), 157.9 (1 C, Ph), 171.6 (1 C, CO). Then, 10d $(0.20 \mathrm{~g}, 0.78 \mathrm{mmol})$ was treated with $\mathrm{LiAlH}_{4}(59 \mathrm{mg}, 1.6 \mathrm{mmol})$ in dry THF $(4.7 \mathrm{~mL})$ to give alcohol intermediate, which was oxidized with PCC $(0.28 \mathrm{~g}, 1.3 \mathrm{mmol})$ in DCM $(6.5 \mathrm{~mL})$ to yield aldehyde 12d $\left(\mathrm{C}_{15} \mathrm{H}_{14} \mathrm{O}_{2}, 0.13 \mathrm{~g}\right.$, $\left.0.58 \mathrm{mmol}\right)$.

2-(3-(3,4-Dichlorophenoxy)phenyl)acetaldehyde (12e). Following the general procedure A, methyl 2-(3-hydroxyphenyl)acetate $(0.60 \mathrm{~g}, 4.4 \mathrm{mmol})$, (3,4-dichlorophenyl)boronic acid $(2.5 \mathrm{~g}$, $13 \mathrm{mmol}), \mathrm{Cu}(\mathrm{OAc})_{2}(1.6 \mathrm{~g}, 8.8 \mathrm{mmol}), 4 \AA$ molecular sieves $(0.79 \mathrm{~g})$ and pyridine $(1.1 \mathrm{~mL}, 13 \mathrm{mmol})$ in 1,2-dichloroethane $(26 \mathrm{~mL})$ afforded the ester intermediate methyl 2-(3-(3,4-dichlorophenoxy)phenyl)acetate 10e (eluent system: $10 \%$ ethylacetate in petroleum ether, $\mathrm{C}_{15} \mathrm{H}_{12} \mathrm{Cl}_{2} \mathrm{O}_{3}, 0.73 \mathrm{~g}, 2.3 \mathrm{mmol}, 53 \%$ yield). ${ }^{1} \mathrm{H}$ NMR $\left(300 \mathrm{MHz}, \mathrm{CDCl}_{3}\right) \delta \mathrm{ppm} 3.63\left(\mathrm{~s}, 2 \mathrm{H}, \mathrm{CH}_{2}\right), 3.71$ $\left(\mathrm{s}, 3 \mathrm{H}, \mathrm{OCH}_{3}\right), 6.86(\mathrm{dd}, J=8.9,2.8 \mathrm{~Hz}, 1 \mathrm{H}, \mathrm{Ph}), 6.90-6.99(\mathrm{~m}, 2 \mathrm{H}, \mathrm{Ph}), 7.06-7.12(\mathrm{~m}, 2 \mathrm{H}, \mathrm{Ph}), 7.31(\mathrm{~d}$, $J=7.9 \mathrm{~Hz}, 1 \mathrm{H}, \mathrm{Ph}), 7.37(\mathrm{~d}, J=8.8 \mathrm{~Hz}, 1 \mathrm{H}, \mathrm{Ph}) .{ }^{13} \mathrm{C} \mathrm{NMR}\left(75 \mathrm{MHz}, \mathrm{CDCl}_{3}\right) \delta \mathrm{ppm} 40.8\left(1 \mathrm{C}, \mathrm{CH}_{2}\right)$, $52.1\left(1 \mathrm{C}, \mathrm{OCH}_{3}\right), 117.8$ (1 C, Ph), 118.0 (1 C, Ph), 120.2 (1 C, Ph), $120.3(1 \mathrm{C}, \mathrm{Ph}), 125.1$ (2 C, Ph), 130.0 (1 C, Ph), 130.9 (1 C, Ph), 133.1 (1 C, Ph), 136.1 (1 C, Ph), 156.1 (1 C, Ph), 156.3 (1 C, Ph), 171.5 (1 C, CO). Then, 10e $(0.20 \mathrm{~g}, 0.64 \mathrm{mmol})$ was treated with $\mathrm{LiAlH}_{4}(49 \mathrm{mg}, 1.3 \mathrm{mmol})$ in dry THF $(3.8 \mathrm{~mL})$ to give alcohol intermediate, which was oxidized with PCC $(0.23 \mathrm{~g}, 1.1 \mathrm{mmol})$ in DCM (5.5 mL) to yield aldehyde $12 \mathbf{e}\left(\mathrm{C}_{14} \mathrm{H}_{10} \mathrm{Cl}_{2} \mathrm{O}_{2}, 0.10 \mathrm{~g}, 0.36 \mathrm{mmol}\right)$.

2-(3-(4-Chlorophenoxy)phenyl)acetaldehyde (12f). Following the general procedure A, methyl 2-(3-hydroxyphenyl)acetate $(0.60 \mathrm{~g}, 4.4 \mathrm{mmol})$, (4-chlorophenyl)boronic acid $(2.1 \mathrm{~g}, 13 \mathrm{mmol}), \mathrm{Cu}(\mathrm{OAc})_{2}$ $(1.6 \mathrm{~g}, 8.8 \mathrm{mmol}), 4 \AA$ molecular sieves $(0.79 \mathrm{~g})$ and pyridine $(1.1 \mathrm{~mL}, 13 \mathrm{mmol})$ in 1,2-dichloroethane $(26 \mathrm{~mL})$ afforded the ester intermediate methyl 2-(3-(4-chlorophenoxy)phenyl)acetate 10f (eluent system: $10 \%$ ethylacetate in petroleum ether, $\mathrm{C}_{15} \mathrm{H}_{13} \mathrm{ClO}_{3}, 0.40 \mathrm{~g}, 1.4 \mathrm{mmol}, 33 \%$ yield). ${ }^{1} \mathrm{H}$ NMR 
$\left(300 \mathrm{MHz}, \mathrm{CDCl}_{3}\right) \delta$ ppm $3.62\left(\mathrm{~s}, 2 \mathrm{H}, \mathrm{CH}_{2}\right), 3.71\left(\mathrm{~s}, 3 \mathrm{H}, \mathrm{OCH}_{3}\right), 6.87-6.99(\mathrm{~m}, 4 \mathrm{H}, \mathrm{Ph}), 7.05(\mathrm{~d}$, $J=7.3 \mathrm{~Hz}, 1 \mathrm{H}, \mathrm{Ph}), 7.25-7.34(\mathrm{~m}, 3 \mathrm{H}, \mathrm{Ph}) .{ }^{13} \mathrm{C} \mathrm{NMR}\left(75 \mathrm{MHz}, \mathrm{CDCl}_{3}\right) \delta \mathrm{ppm} 40.9\left(1 \mathrm{C} \mathrm{CH}_{2}\right), 52.1(1$ C, $\left.\mathrm{OCH}_{3}\right), 117.4$ (1 C, Ph), 119.8 (1 C, Ph), 120.2 (2 C, Ph), 124.5 (1 C, Ph), 128.4 (1 C, Ph), 129.8 (2 C, Ph), 129.9 (1 C, Ph), 136.0 (1 C, Ph), 155.7 (1 C, Ph), 157.1 (1 C, Ph), 171.6 (1 C, CO). Then, $10 f(0.20$ g, 0.72 mmol) was treated with $\mathrm{LiAlH}_{4}(55 \mathrm{mg}, 1.5 \mathrm{mmol})$ in dry THF $(4.3 \mathrm{~mL})$ to give alcohol intermediate, which was oxidized with PCC $(0.31 \mathrm{~g}, 1.5 \mathrm{mmol})$ in DCM $(7.5 \mathrm{~mL})$ to yield aldehyde $\mathbf{1 2 f}\left(\mathrm{C}_{14} \mathrm{H}_{11} \mathrm{ClO}_{2}\right.$, $0.13 \mathrm{~g}, 0.53 \mathrm{mmol})$.

2-(3-(4-Methoxyphenoxy)phenyl)acetaldehyde (12g). Following the general procedure A, methyl 2-(3-hydroxyphenyl)acetate $(0.60 \mathrm{~g}, 4.4 \mathrm{mmol})$, (4-methoxyphenyl)boronic acid $(2.0 \mathrm{~g}$, $13 \mathrm{mmol}), \mathrm{Cu}(\mathrm{OAc})_{2}(1.6 \mathrm{~g}, 8.8 \mathrm{mmol}), 4 \AA$ molecular sieves $(0.79 \mathrm{~g})$ and pyridine $(1.1 \mathrm{~mL}, 13 \mathrm{mmol})$ in 1,2-dichloroethane $(26 \mathrm{~mL})$ afforded the ester intermediate methyl 2-(3-(4-methoxyphenoxy)phenyl)acetate $10 \mathrm{~g}$ (eluent system: $10 \%$ ethylacetate in petroleum ether, $\mathrm{C}_{16} \mathrm{H}_{16} \mathrm{O}_{4}, 0.33 \mathrm{~g}, 1.2 \mathrm{mmol}, 28 \%$ yield). ${ }^{1} \mathrm{H}$ NMR $\left(300 \mathrm{MHz}, \mathrm{CDCl}_{3}\right) \delta \mathrm{ppm} 3.60\left(\mathrm{~s}, 2 \mathrm{H}, \mathrm{CH}_{2}\right), 3.70$ (s, 3 $\left.\mathrm{H}, \mathrm{OCH}_{3}\right), 3.82\left(\mathrm{~s}, 3 \mathrm{H},(\mathrm{Ph}) \mathrm{OCH}_{3}\right), 6.82-6.87(\mathrm{~m}, 1 \mathrm{H}, \mathrm{Ph}), 6.88-6.94(\mathrm{~m}, 3 \mathrm{H}, \mathrm{Ph}), 6.95-7.03(\mathrm{~m}, 3 \mathrm{H}$, $\mathrm{Ph}), 7.22-7.29(\mathrm{~m}, 1 \mathrm{H}, \mathrm{Ph}) .{ }^{13} \mathrm{C} \mathrm{NMR}\left(75 \mathrm{MHz}, \mathrm{CDCl}_{3}\right) \delta \mathrm{ppm} 41.0\left(1 \mathrm{C}, \mathrm{CH}_{2}\right), 52.0\left(1 \mathrm{C}, \mathrm{OCH}_{3}\right), 55.6(1$ C, $\left.(\mathrm{Ph}) \mathrm{OCH}_{3}\right), 114.8$ (2 C, Ph), 116.0 (1 C, Ph), 118.4 (1 C, Ph), 120.9 (2 C, Ph), 123.2 (1 C, Ph), 129.6 (1 C, Ph), 135.6 (1 C, Ph), 149.8 (1 C, Ph), 155.9 (1 C, Ph), 158.6 (1 C, Ph), 171.7 (1 C, CO). Then, 10g $(0.20 \mathrm{~g}, 0.74 \mathrm{mmol})$ was treated with $\mathrm{LiAlH}_{4}(56 \mathrm{mg}, 1.5 \mathrm{mmol})$ in dry THF $(4.4 \mathrm{~mL})$ to give alcohol intermediate, which was oxidized with PCC $(0.32 \mathrm{~g}, 1.5 \mathrm{mmol})$ in DCM $(7.5 \mathrm{~mL})$ to yield aldehyde $\mathbf{1 2} \mathrm{g}$ $\left(\mathrm{C}_{15} \mathrm{H}_{14} \mathrm{O}_{3}, 0.14 \mathrm{~g}, 0.58 \mathrm{mmol}\right)$.

2-(3-(3-Chlorophenoxy)phenyl)acetaldehyde (12h). Following the general procedure A, methyl 2-(3-hydroxyphenyl)acetate $(0.40 \mathrm{~g}, 2.9 \mathrm{mmol}), 3$-chlorophenylboronic acid $(1.4 \mathrm{~g}, 8.8 \mathrm{mmol}), \mathrm{Cu}(\mathrm{OAc})_{2}$ $(1.1 \mathrm{~g}, 5.9 \mathrm{mmol}), 4 \AA$ molecular sieves $(0.50 \mathrm{~g})$ and pyridine $(0.71 \mathrm{~mL}, 8.8 \mathrm{mmol})$ in 1,2-dichloroethane $(17 \mathrm{~mL})$ afforded the ester intermediate methyl 2-(3-(3-chlorophenoxy)phenyl)acetate 10h (eluent system: $10 \%$ ethylacetate in petroleum ether, $\mathrm{C}_{15} \mathrm{H}_{13} \mathrm{ClO}_{3}, 0.28 \mathrm{~g}, 1.0 \mathrm{mmol}, 34 \%$ yield). ${ }^{1} \mathrm{H}$ NMR $\left(300 \mathrm{MHz}, \mathrm{CDCl}_{3}\right) \delta \mathrm{ppm} 3.63\left(\mathrm{~s}, 2 \mathrm{H}, \mathrm{CH}_{2}\right), 3.71\left(\mathrm{~s}, 3 \mathrm{H}, \mathrm{OCH}_{3}\right), 6.88-6.96(\mathrm{~m}, 2 \mathrm{H}, \mathrm{Ph}), 6.98(\mathrm{t}, J=1.9 \mathrm{~Hz}$, $1 \mathrm{H}, \mathrm{Ph}), 7.00(\mathrm{t}, J=2.2 \mathrm{~Hz}, 1 \mathrm{H}, \mathrm{Ph}), 7.04-7.14(\mathrm{~m}, 2 \mathrm{H}, \mathrm{Ph}), 7.17-7.36(\mathrm{~m}, 2 \mathrm{H}, \mathrm{Ph}) .{ }^{13} \mathrm{C}$ NMR $(75 \mathrm{MHz}$, $\left.\mathrm{CDCl}_{3}\right) \delta$ ppm $40.9\left(1 \mathrm{C}, \mathrm{CH}_{2}\right), 52.1\left(1 \mathrm{C}, \mathrm{OCH}_{3}\right), 116.8$ (1 C, Ph), 117.9 (1 C, Ph), 118.9 (1 C, Ph), 120.2 (1 C, Ph), 123.3 (1 C, Ph), 124.8 (1 C, Ph), 130.0 (1 C, Ph), 130.5 (1 C, Ph), 135.0 (1 C, Ph), 136.0 (1 C, Ph), 156.5 (1 C, Ph), 158.1 (1 C, Ph), 171.5 (1 C, CO). Then, $10 \mathrm{~h}(0.20 \mathrm{~g}, 0.72 \mathrm{mmol})$ was treated with $\mathrm{LiAlH}_{4}$ (55 mg, $1.4 \mathrm{mmol})$ in dry THF $(4.3 \mathrm{~mL})$ to give alcohol intermediate, which was oxidized with PCC $(0.19 \mathrm{~g}, 0.87 \mathrm{mmol})$ in DCM $(4.3 \mathrm{~mL})$ to yield aldehyde $\mathbf{1 2 h}\left(\mathrm{C}_{14} \mathrm{H}_{11} \mathrm{ClO}_{2}, 0.10 \mathrm{~g}, 0.41 \mathrm{mmol}\right)$.

2-(3-(Benzyloxy)phenyl)acetaldehyde (15a). According to a literature procedure [27] with minor changes, methyl 2-(3-hydroxyphenyl)acetate $(0.30 \mathrm{~g}, 2.2 \mathrm{mmol})$, benzyl bromide $(0.26 \mathrm{~mL}, 2.2 \mathrm{mmol}), \mathrm{K}_{2} \mathrm{CO}_{3}$ $(0.61 \mathrm{~g}, 4.4 \mathrm{mmol})$, sodium iodide (33 $\mathrm{mg}, 0.22 \mathrm{mmol})$ in dimethylformamide (DMF) $(10 \mathrm{~mL})$ at room temperature for overnight afforded the ester intermediate methyl 2-(3-(benzyloxy)phenyl)acetate 13a (eluent system: $10 \%$ ethylacetate in petroleum ether, $\mathrm{C}_{16} \mathrm{H}_{16} \mathrm{O}_{3}, 0.33 \mathrm{~g}, 1.3 \mathrm{mmol}, 58 \%$ yield). ${ }^{1} \mathrm{H}$ NMR $\left(300 \mathrm{MHz}, \mathrm{CDCl}_{3}\right) \delta \mathrm{ppm} 3.65\left(\mathrm{~s}, 2 \mathrm{H}, \mathrm{CH}_{2}\right), 3.73\left(\mathrm{~s}, 3 \mathrm{H}, \mathrm{OCH}_{3}\right), 5.10\left(\mathrm{~s}, 2 \mathrm{H},\left(\mathrm{Ph}^{2} \mathrm{CH}_{2} \mathrm{O}\right), 6.83-7.05(\mathrm{~m}\right.$, $3 \mathrm{H}, \mathrm{Ph}), 7.21-7.52(\mathrm{~m}, 6 \mathrm{H}, \mathrm{Ph}) .{ }^{13} \mathrm{C} \mathrm{NMR}\left(75 \mathrm{MHz}, \mathrm{CDCl}_{3}\right) \delta \mathrm{ppm} 41.1\left(1 \mathrm{C}, \mathrm{CH}_{2}\right), 52.0\left(1 \mathrm{C} \mathrm{OCH}_{3}\right)$, 69.8 (1 C, $\left.(\mathrm{Ph}) \mathrm{CH}_{2} \mathrm{O}\right), 113.4$ (1 C, Ph), 115.8 (1 C, Ph), 121.8 (1 C, Ph), 127.5 (2 C, Ph), 127.9 (1 C, Ph), 128.5 (2 C, Ph), 129.5 (1 C, Ph), 135.4 (1 C, Ph), 136.9 (1 C, Ph), 158.9 (1 C, Ph), 171.8 (1 C, CO). Then, 13a $(0.20 \mathrm{~g}, 0.78 \mathrm{mmol})$ was treated with $\mathrm{LiAlH}_{4}(59 \mathrm{mg}, 1.6 \mathrm{mmol})$ in dry THF $(4.7 \mathrm{~mL})$ to give alcohol intermediate, which was oxidized with PCC $(0.34 \mathrm{~g}, 1.6 \mathrm{mmol})$ in DCM $(8.0 \mathrm{~mL})$ to yield aldehyde 15a $\left(\mathrm{C}_{15} \mathrm{H}_{14} \mathrm{O}_{2}, 0.14 \mathrm{~g}, 0.62 \mathrm{mmol}\right)$.

2-(3-((2-Chlorobenzyl)oxy)phenyl)acetaldehyde (15b). Following the procedure as described for 15a, methyl 2-(3-hydroxyphenyl)acetate $(0.40 \mathrm{~g}, 2.9 \mathrm{mmol}), 1$-(bromomethyl)-2-chlorobenzene $(0.60 \mathrm{~g}, 2.9 \mathrm{mmol})$, $\mathrm{K}_{2} \mathrm{CO}_{3}(0.81 \mathrm{~g}, 5.9 \mathrm{mmol})$, sodium iodide $(44 \mathrm{mg}, 0.29 \mathrm{mmol})$ in DMF $(13 \mathrm{~mL})$ afforded the ester intermediate methyl 2-(3-((2-chlorobenzyl)oxy)phenyl)acetate $\mathbf{1 3 b}$ (eluent system: 10\% ethylacetate in 
petroleum ether, $\mathrm{C}_{16} \mathrm{H}_{15} \mathrm{ClO}_{3}, 0.26 \mathrm{~g}, 0.89 \mathrm{mmol}, 30 \%$ yield). ${ }^{1} \mathrm{H}$ NMR (300 MHz, $\left.\mathrm{CDCl}_{3}\right) \delta \mathrm{ppm} 3.63$ $\left(\mathrm{s}, 2 \mathrm{H}, \mathrm{CH}_{2}\right), 3.71\left(\mathrm{~s}, 3 \mathrm{H}, \mathrm{OCH}_{3}\right), 5.17\left(\mathrm{~s}, 2 \mathrm{H},(\mathrm{Ph}) \mathrm{CH}_{2} \mathrm{O}\right), 6.86-7.01(\mathrm{~m}, 3 \mathrm{H}, \mathrm{Ph}), 7.23-7.36(\mathrm{~m}, 3 \mathrm{H}$, $\mathrm{Ph}), 7.41(\mathrm{dd}, J=7.0,2.1 \mathrm{~Hz}, 1 \mathrm{H}, \mathrm{Ph}), 7.53-7.63(\mathrm{~m}, 1 \mathrm{H}, \mathrm{Ph}) .{ }^{13} \mathrm{C}$ NMR $\left(75 \mathrm{MHz}, \mathrm{CDCl}_{3}\right) \delta \mathrm{ppm} 41.2(1$ $\left.\mathrm{C}, \mathrm{CH}_{2}\right), 52.1\left(1 \mathrm{C}, \mathrm{OCH}_{3}\right), 67.1\left(1 \mathrm{C},(\mathrm{Ph}) \mathrm{CH}_{2} \mathrm{O}\right), 113.4(1 \mathrm{C}, \mathrm{Ph}), 116.0(1 \mathrm{C}, \mathrm{Ph}), 122.1(1 \mathrm{C}, \mathrm{Ph}), 126.9$ (1 C, Ph), 128.8 (1 C, Ph), 129.0 (1 C, Ph), 129.3 (1 C, Ph), 129.6 (1 C, Ph), 132.6 (1 C, Ph), 134.7 (1 C, Ph), 135.5 (1 C, Ph), 158.7 (1 C, Ph), 171.8 (1 C, CO). Then, 13b $(0.20 \mathrm{~g}, 0.69 \mathrm{mmol})$ was treated with $\mathrm{LiAlH}_{4}$ (52 mg, $1.4 \mathrm{mmol})$ in dry THF ( $4.1 \mathrm{~mL})$ to give alcohol intermediate, which was oxidized with PCC $(0.25 \mathrm{~g}, 1.2 \mathrm{mmol})$ in DCM $(6.0 \mathrm{~mL})$ to yield aldehyde $\mathbf{1 5 b}\left(\mathrm{C}_{15} \mathrm{H}_{13} \mathrm{ClO}_{2}, 0.14 \mathrm{~g}, 0.53 \mathrm{mmol}\right)$.

2-(3-((3,4-Dichlorobenzyl)oxy) phenyl)acetaldehyde (15c). Following the procedure as described for 15a, methyl 2-(3-hydroxyphenyl)acetate $(0.40 \mathrm{~g}, 2.9 \mathrm{mmol})$, 3,4-dichlorobenzyl bromide ( $0.70 \mathrm{~g}, 2.9 \mathrm{mmol})$, $\mathrm{K}_{2} \mathrm{CO}_{3}(0.81 \mathrm{~g}, 5.9 \mathrm{mmol})$, sodium iodide $(44 \mathrm{mg}, 0.29 \mathrm{mmol})$ in DMF $(13 \mathrm{~mL})$ afforded the ester intermediate methyl 2-(3-((3,4-dichlorobenzyl)oxy)phenyl)acetate 13c (eluent system: 10\% ethylacetate in petroleum ether, $\mathrm{C}_{16} \mathrm{H}_{14} \mathrm{Cl}_{2} \mathrm{O}_{3}, 0.75 \mathrm{~g}, 2.3 \mathrm{mmol}, 84 \%$ yield). ${ }^{1} \mathrm{H}$ NMR $\left(300 \mathrm{MHz}, \mathrm{CDCl}_{3}\right) \delta \mathrm{ppm} 3.62$ $\left(\mathrm{s}, 2 \mathrm{H}, \mathrm{CH}_{2}\right), 3.70\left(\mathrm{~s}, 3 \mathrm{H}, \mathrm{OCH}_{3}\right), 5.01\left(\mathrm{~s}, 2 \mathrm{H},(\mathrm{Ph}) \mathrm{CH}_{2} \mathrm{O}\right), 6.87(\mathrm{~d}, J=8.2 \mathrm{~Hz}, 1 \mathrm{H}, \mathrm{Ph}), 6.93$ (br. s., $2 \mathrm{H}$, $\mathrm{Ph}), 7.21-7.30(\mathrm{~m}, 2 \mathrm{H}, \mathrm{Ph}), 7.41-7.48(\mathrm{~m}, 1 \mathrm{H}, \mathrm{Ph}), 7.54(\mathrm{~s}, 1 \mathrm{H}, \mathrm{Ph}) .{ }^{13} \mathrm{C} \mathrm{NMR}\left(75 \mathrm{MHz}, \mathrm{CDCl}_{3}\right) \delta \mathrm{ppm}$ $41.1\left(1 \mathrm{C}, \mathrm{CH}_{2}\right), 52.1\left(1 \mathrm{C}, \mathrm{OCH}_{3}\right), 68.4\left(1 \mathrm{C},(\mathrm{Ph}) \mathrm{CH}_{2} \mathrm{O}\right), 113.4(1 \mathrm{C}, \mathrm{Ph}), 115.8(1 \mathrm{C}, \mathrm{Ph}), 122.3(1 \mathrm{C}, \mathrm{Ph})$, 126.5 (1 C, Ph), 129.2 (1 C, Ph), 129.7 (1 C, Ph), 130.5 (1 C, Ph), 131.8 (1 C, Ph), 132.6 (1 C, Ph), 135.6 (1 C, $\mathrm{Ph}), 137.3$ (1 C, Ph), 158.4 (1 C, Ph), 171.7 (1 C, CO). Then, 13c $(0.20 \mathrm{~g}, 0.62 \mathrm{mmol})$ was treated with $\mathrm{LiAlH}_{4}(47 \mathrm{mg}, 1.2 \mathrm{mmol})$ in dry THF $(3.7 \mathrm{~mL})$ to give alcohol intermediate, which was oxidized with PCC (0.26 g, $1.2 \mathrm{mmol})$ in DCM $(6.0 \mathrm{~mL})$ to yield aldehyde $15 \mathrm{c}\left(\mathrm{C}_{15} \mathrm{H}_{12} \mathrm{Cl}_{2} \mathrm{O}_{2}, 0.18 \mathrm{~g}\right.$, $\left.0.60 \mathrm{mmol}\right)$.

2-(3-((3-Chlorobenzyl)oxy)phenyl)acetaldehyde (15d). Following the procedure as described for 15a, methyl 2-(3-hydroxyphenyl)acetate $(0.40 \mathrm{~g}, 2.9 \mathrm{mmol})$, m-chlorobenzyl bromide $(0.60 \mathrm{~g}, 2.9 \mathrm{mmol}), \mathrm{K}_{2} \mathrm{CO}_{3}$ $(0.81 \mathrm{~g}, 5.9 \mathrm{mmol})$, sodium iodide $(44 \mathrm{mg}, 0.29 \mathrm{mmol})$ in DMF $(13 \mathrm{~mL})$ afforded the ester intermediate methyl 2-(3-((3-chlorobenzyl)oxy)phenyl)acetate 13d (eluent system: $10 \%$ ethylacetate in petroleum ether, $\mathrm{C}_{16} \mathrm{H}_{15} \mathrm{ClO}_{3}, 0.62 \mathrm{~g}, 2.1 \mathrm{mmol}, 73 \%$ yield). ${ }^{1} \mathrm{H}$ NMR $\left(400 \mathrm{MHz}, \mathrm{CDCl}_{3}\right) \delta \mathrm{ppm} 3.61\left(\mathrm{~s}, 2 \mathrm{H}, \mathrm{CH}_{2}\right)$, $3.70\left(\mathrm{~s}, 3 \mathrm{H}, \mathrm{OCH}_{3}\right), 5.03\left(\mathrm{~s}, 2 \mathrm{H},(\mathrm{Ph}) \mathrm{CH}_{2} \mathrm{O}\right), 6.84-6.94(\mathrm{~m}, 3 \mathrm{H}, \mathrm{Ph}), 7.23-7.28(\mathrm{~m}, 1 \mathrm{H}, \mathrm{Ph}), 7.29-7.32(\mathrm{~m}$, $3 \mathrm{H}, \mathrm{Ph}), 7.35-7.48(\mathrm{~m}, 1 \mathrm{H}, \mathrm{Ph}) .{ }^{13} \mathrm{C}$ NMR $\left(101 \mathrm{MHz}, \mathrm{CDCl}_{3}\right) \delta \mathrm{ppm} 41.2\left(1 \mathrm{C}, \mathrm{CH}_{2}\right), 52.1\left(1 \mathrm{C}, \mathrm{OCH}_{3}\right)$, 69.1 (1 C, $\left.(\mathrm{Ph}) \mathrm{CH}_{2} \mathrm{O}\right), 113.4$ (1 C, Ph), 115.8 (1 C, Ph), 122.1 (1 C, Ph), 125.3 (1 C, Ph), $127.4(1 \mathrm{C}, \mathrm{Ph})$, 128.0 (1 C, Ph), 129.6 (1 C, Ph), 129.8 (1 C, Ph), 134.5 (1 C, Ph), 135.5 (1 C, Ph), 139.0 (1 C, Ph), 158.6 (1 C, $\mathrm{Ph}), 171.9(1 \mathrm{C}, \mathrm{CO})$. Then, $13 \mathrm{~d}(0.20 \mathrm{~g}, 0.69 \mathrm{mmol})$ was treated with $\mathrm{LiAlH}_{4}(52 \mathrm{mg}, 1.4 \mathrm{mmol})$ in dry THF (4.1 mL) to give alcohol intermediate, which was oxidized with PCC $(0.26 \mathrm{~g}, 1.2 \mathrm{mmol})$ in DCM (6.0 mL) to yield aldehyde $15 \mathrm{~d}\left(\mathrm{C}_{15} \mathrm{H}_{13} \mathrm{ClO}_{2}, 0.14 \mathrm{~g}, 0.52 \mathrm{mmol}\right)$.

2-(3-(4-Cyanophenoxy)phenyl)acetaldehyde (18a). To a solution of methyl 2-(3-hydroxyphenyl)acetate (0.35 g, $2.1 \mathrm{mmol})$ in THF $(13 \mathrm{~mL})$ was added $\mathrm{LiAlH}_{4}(0.16 \mathrm{~g}$, $4.2 \mathrm{mmol})$ afforded 3-(2-hydroxyethyl)phenol $(0.24 \mathrm{~g}, 1.4 \mathrm{mmol})$, which was reacted with 4-fluorobenzonitrile $(0.21 \mathrm{~g}, 1.7 \mathrm{mmol}), \mathrm{K}_{2} \mathrm{CO}_{3}(0.40 \mathrm{~g}$, $2.9 \mathrm{mmol})$ in DMF $(10 \mathrm{~mL})$ at $90{ }^{\circ} \mathrm{C}$ gave 4-(3-(2-hydroxyethyl)phenoxy)benzonitrile [28] 17a (eluent system: $25 \%$ ethylacetate in petroleum ether, $\mathrm{C}_{15} \mathrm{H}_{13} \mathrm{NO}_{2}, 0.14 \mathrm{~g}, 0.59 \mathrm{mmol}, 40 \%$ yield). ${ }^{1} \mathrm{H}$ NMR $\left(300 \mathrm{MHz}, \mathrm{CDCl}_{3}\right) \delta$ ppm $2.89\left(\mathrm{t}, J=6.6 \mathrm{~Hz}, 2 \mathrm{H}, \mathrm{CH}_{2}\right), 3.89\left(\mathrm{t}, J=6.4 \mathrm{~Hz}, 2 \mathrm{H}, \mathrm{CH}_{2}(\mathrm{OH})\right), 6.89-7.06(\mathrm{~m}, 4$ $\mathrm{H}, \mathrm{Ph}), 7.11(\mathrm{dd}, J=7.6,0.59 \mathrm{~Hz}, 1 \mathrm{H}, \mathrm{Ph}), 7.36(\mathrm{t}, J=7.4 \mathrm{~Hz}, 1 \mathrm{H}, \mathrm{Ph}), 7.49-7.69(\mathrm{~m}, 2 \mathrm{H}, \mathrm{Ph}) .{ }^{13} \mathrm{C} \mathrm{NMR}$ $\left(75 \mathrm{MHz}, \mathrm{CDCl}_{3}\right)$ Sppm 38.9 (1 C, $\left.\mathrm{CH}_{2}\right), 63.3\left(1 \mathrm{C}, \mathrm{CH}_{2}(\mathrm{OH})\right), 105.8$ (1 C, Ph), 110.0 (1 C, Ph), 118.0 (2 C, Ph), 118.3 (1 C, CN), 120.9 (1 C, Ph), 125.7 (1 C, Ph), 130.2 (1 C, Ph), 134.1 (2 C, Ph), 141.4 (1 C, Ph), 154.9 (1 C, Ph), 161.5 (1 C, Ph). Then, 17a was oxidized by PCC $(0.25 \mathrm{~g}, 1.2 \mathrm{mmol})$ in DCM (6.0 $\mathrm{mL})$ to give aldehyde $18 \mathbf{a}\left(\mathrm{C}_{15} \mathrm{H}_{11} \mathrm{NO}_{2}, 0.11 \mathrm{~g}, 0.44 \mathrm{mmol}\right)$.

2-(3-(4-Nitrophenoxy)phenyl)acetaldehyde (18b). Following the procedure described for 18a, 3-(2-hydroxyethyl)phenol $(0.30 \mathrm{~g}, 2.2 \mathrm{mmol})$, 1-fluoro-4-nitrobenzene $(0.37 \mathrm{~g}, 2.6 \mathrm{mmol})$ and $\mathrm{K}_{2} \mathrm{CO}_{3}$ (0.60 g, $4.3 \mathrm{mmol})$ in DMF (16 mL) afforded 2-(3-(4-nitrophenoxy)phenyl)ethan-1-ol 17b (eluent system: $25 \%$ ethylacetate in petroleum ether, $\mathrm{C}_{14} \mathrm{H}_{13} \mathrm{NO}_{4}, 0.52 \mathrm{~g}, 2.0 \mathrm{mmol}, 92 \%$ yield). ${ }^{1} \mathrm{H}$ NMR (300 MHz, $\left.\mathrm{CDCl}_{3}\right) \delta$ ppm $2.90\left(\mathrm{t}, J=6.4 \mathrm{~Hz}, 2 \mathrm{H}, \mathrm{CH}_{2}\right), 3.89\left(\mathrm{t}, J=6.4 \mathrm{~Hz}, 2 \mathrm{H}, \mathrm{CH}_{2}(\mathrm{OH})\right), 6.93-7.07(\mathrm{~m}, 4 \mathrm{H}, \mathrm{Ph})$, 
$7.13(\mathrm{~d}, J=7.6 \mathrm{~Hz}, 1 \mathrm{H}, \mathrm{Ph}), 7.37(\mathrm{t}, J=7.7 \mathrm{~Hz}, 1 \mathrm{H}, \mathrm{Ph}), 8.13-8.24(\mathrm{~m}, 2 \mathrm{H}, \mathrm{Ph}) .{ }^{13} \mathrm{C} \mathrm{NMR}(75 \mathrm{MHz}$, $\left.\mathrm{CDCl}_{3}\right) \delta$ ppm $38.8\left(1 \mathrm{C}, \mathrm{CH}_{2}\right), 63.2\left(1 \mathrm{C}, \mathrm{CH}_{2}(\mathrm{OH})\right), 117.1(2 \mathrm{C}, \mathrm{Ph}), 118.4(1 \mathrm{C}, \mathrm{Ph}), 121.0(1 \mathrm{C}, \mathrm{Ph})$, 125.9 (2 C, Ph), 126.0 (1 C, Ph), 130.3 (1 C, Ph), 136.5 (1 C, Ph), 141.5 (1 C, Ph), 154.9 (1 C, Ph), 163.2 $(1 \mathrm{C}, \mathrm{Ph})$. Then, $\mathbf{1 7 b}$ was oxidized by PCC $(0.86 \mathrm{~g}, 4.0 \mathrm{mmol})$ in DCM $(20 \mathrm{~mL})$ to give aldehyde $\mathbf{1 8 b}$ $\left(\mathrm{C}_{14} \mathrm{H}_{11} \mathrm{NO}_{4}, 0.36 \mathrm{~g}, 1.4 \mathrm{mmol}\right)$.

2-(3-(4-(Trifluoromethyl)phenoxy)phenyl)acetaldehyde (18c). Following the procedure described for 18a, 3-(2-hydroxyethyl)phenol $(0.30 \mathrm{~g}, 2.2 \mathrm{mmol})$, 1-fluoro-4-(trifluoromethyl)benzene $(0.43 \mathrm{~g}, 2.6 \mathrm{mmol})$ and $\mathrm{CsCO}_{3}(0.85 \mathrm{~g}, 2.6 \mathrm{mmol})$ in $\mathrm{DMF}(16 \mathrm{~mL})$ at $90{ }^{\circ} \mathrm{C}$ gave 2-(3-(4-(trifluoromethyl)phenoxy)phenyl)ethan-1-ol 17c (eluent system: $25 \%$ ethylacetate in petroleum ether, $\mathrm{C}_{15} \mathrm{H}_{13} \mathrm{~F}_{3} \mathrm{O}_{2}, 0.13 \mathrm{~g}, 0.46 \mathrm{mmol}, 21 \%$ yield). ${ }^{1} \mathrm{H}$ NMR $\left(300 \mathrm{MHz}, \mathrm{CDCl}_{3}\right) \delta \mathrm{ppm} 2.87(\mathrm{t}, J=6.6 \mathrm{~Hz}$, $\left.2 \mathrm{H}, \mathrm{CH}_{2}\right), 3.86\left(\mathrm{t}, J=6.6 \mathrm{~Hz}, 2 \mathrm{H}, \mathrm{CH}_{2}(\mathrm{OH})\right), 6.89-6.98(\mathrm{~m}, 2 \mathrm{H}, \mathrm{Ph}), 7.01-7.12(\mathrm{~m}, 3 \mathrm{H}, \mathrm{Ph}), 7.33(\mathrm{t}$, $J=6.6 \mathrm{~Hz}, 1 \mathrm{H}, \mathrm{Ph}), 7.58(\mathrm{~d}, J=8.8 \mathrm{~Hz}, 2 \mathrm{H}, \mathrm{Ph}) .{ }^{13} \mathrm{C} \mathrm{NMR}\left(75 \mathrm{MHz}, \mathrm{CDCl}_{3}\right) \delta \mathrm{ppm} 38.9\left(1 \mathrm{C}, \mathrm{CH}_{2}\right)$, $63.3\left(1 \mathrm{C}, \mathrm{CH}_{2}(\mathrm{OH})\right), 117.7(1 \mathrm{C}, \mathrm{Ph}), 117.8(2 \mathrm{C}, \mathrm{Ph}), 120.4(2 \mathrm{C}, \mathrm{Ph}), 124.2\left(\mathrm{q}, J=271.5 \mathrm{~Hz}, 1 \mathrm{C}, \mathrm{CF}_{3}\right)$, 125.1 (1 C, Ph), 127.0 (2 C, Ph), 130.0 (1 C, Ph), 141.1 (1 C, Ph), 155.9 (1 C, Ph), 160.3 (1 C, Ph). Then, 17c was oxidized by PCC $(0.20 \mathrm{~g}, 0.92 \mathrm{mmol})$ in DCM $(4.6 \mathrm{~mL})$ to give aldehyde $18 \mathrm{c}\left(\mathrm{C}_{15} \mathrm{H}_{11} \mathrm{~F}_{3} \mathrm{O}_{2}, 0.12 \mathrm{~g}\right.$, $0.43 \mathrm{mmol})$.

5-Methyl-1-(1-phenethylpiperidin-4-yl)pyrimidine-2,4(1H,3H)-dione (21a). Following the general procedure $\mathrm{B}$, phenylacetaldehyde $(36 \mathrm{mg}, 0.30 \mathrm{mmol}), 28(0.10 \mathrm{~g}, 0.30 \mathrm{mmol})$ and sodium triacetoxyborohydride $(0.13 \mathrm{~g}, 0.61 \mathrm{mmol})$ in dichloroethane $(10 \mathrm{~mL})$ afforded the BOM-protected intermediate, which was deprotected with TFA $(5.0 \mathrm{~mL})$ in the presence of triethylsilane $(5.0 \mathrm{~mL})$ at $73{ }^{\circ} \mathrm{C}$ for $4 \mathrm{~h}$ to give the $\mathbf{2 1 a}$ (eluent system: $5 \% \mathrm{MeOH}$ in DCM, $53 \mathrm{mg}, 0.17 \mathrm{mmol}, 56 \%$ yield). ${ }^{1} \mathrm{H}$ NMR (300 MHz, DMSO-d $\mathrm{d}_{6}$ ) $\delta$ ppm 1.53-1.93 (m, $7 \mathrm{H}, 5-\mathrm{CH}_{3}$, piperdyl-3-yl, piperidyl-5-yl), 2.00-2.16 (m, $2 \mathrm{H}$, piperidyl-2a-yl, piperidyl-6a-yl), $2.57\left(\mathrm{~d}, J=8.2 \mathrm{~Hz}, 2 \mathrm{H}, \mathrm{CH}_{2} \mathrm{~N}\right), 2.66-2.82\left(\mathrm{~m}, 2 \mathrm{H}, \mathrm{PhCH}_{2}\right), 3.06(\mathrm{~d}, J=10.8 \mathrm{~Hz}, 2 \mathrm{H}$, piperidyl-2b-yl, piperidyl-6b-yl), 4.16-4.36 (m, $1 \mathrm{H}$, piperidyl-4-yl), 7.10-7.37 (m, $5 \mathrm{H}, \mathrm{Ph}), 7.63(\mathrm{~s}, 1 \mathrm{H}$, H-6), 11.19 (s, 1 H, NH). ${ }^{13} \mathrm{C}$ NMR (75 MHz, DMSO-d 6 ) $\delta$ ppm 12.0 (1 C, 5- $\left.\mathrm{CH}_{3}\right), 29.9$ (2 C, piperdyl-3-yl, piperidyl-5-yl), 33.0 (1 C, $\left.\mathrm{PhCH}_{2}\right), 52.4$ (2 C, piperidyl-2-yl, piperidyl-6-yl), 52.6 (1 C, piperidyl-4-yl), $59.3\left(1 \mathrm{C}, \mathrm{CH}_{2} \mathrm{~N}\right), 108.9$ (1 C, C-5), 125.8 (1 C, Ph), 128.2 (2 C, Ph), 128.6 (2 C, Ph), 137.7 (1 C, C-6), 140.4 (1 C, Ph), 150.8 (1 C, C-2), 163.7 (1 C, C-4). HRMS (ESI): $m / z[\mathrm{M}+\mathrm{H}]^{+}$Calcd. for $\left[\mathrm{C}_{18} \mathrm{H}_{23} \mathrm{~N}_{3} \mathrm{O}_{2}+\mathrm{H}\right]^{+}$ 314.1863, found 314.1855.

1-(1-(2-([1,1'-Biphenyl]-4-yl)ethyl)piperidin-4-yl)-5-methylpyrimidine-2,4(1H,3H)-dione (21b). Following the general procedure $B, 6(60 \mathrm{mg}, 0.30 \mathrm{mmol}), 28(0.10 \mathrm{~g}, 0.30 \mathrm{mmol})$ and sodium triacetoxyborohydride $(0.13 \mathrm{~g}, 0.61 \mathrm{mmol})$ in dichloroethane $(10 \mathrm{~mL})$ afforded the BOM-protected intermediate, which was deprotected with TFA $(5.0 \mathrm{~mL})$ in the presence of triethylsilane $(5.0 \mathrm{~mL})$ at $73{ }^{\circ} \mathrm{C}$ for $4 \mathrm{~h}$ to give the $\mathbf{2 1 b}$ (eluent system: $5 \% \mathrm{MeOH}$ in DCM, $39 \mathrm{mg}, 0.10 \mathrm{mmol}, 33 \%$ yield). ${ }^{1} \mathrm{H}$ NMR (400 MHz, DMSO- $\mathrm{d}_{6}$ ) $\delta$ ppm 1.72-1.93 (m, $5 \mathrm{H}$, piperdyl-3a-yl, piperidyl-5a-yl, 5- $\left.\mathrm{CH}_{3}\right), 1.98-2.20(\mathrm{~m}, 2 \mathrm{H}$, piperdyl-3b-yl, piperidyl-5b-yl), 2.89-3.04 (m, $\left.2 \mathrm{H}, \mathrm{PhCH}_{2}\right), 3.26-3.61$ (m, $4 \mathrm{H}$, piperidyl-2-yl, piperidyl-6-yl), 4.39-4.53 (m, $1 \mathrm{H}$, piperidyl-4-yl), 7.33-7.40 (m, $3 \mathrm{H}, \mathrm{Ph}), 7.46(\mathrm{t}, J=7.6 \mathrm{~Hz}, 2 \mathrm{H}, \mathrm{Ph}), 7.59-7.71(\mathrm{~m}, 5 \mathrm{H}, \mathrm{H}-6$, $\mathrm{Ph}), 11.30(\mathrm{~s}, 1 \mathrm{H}, \mathrm{NH}), 2 \mathrm{H}\left(\mathrm{CH}_{2} \mathrm{~N}\right)$ could not be observed. ${ }^{13} \mathrm{C} \mathrm{NMR}\left(101 \mathrm{MHz}, \mathrm{DMSO}-\mathrm{d}_{6}\right) \delta \mathrm{ppm}$ 12.1 (1 C, 5- $\left.\mathrm{CH}_{3}\right), 27.7$ (2 C, piperdyl-3-yl, piperidyl-5-yl), 29.9 (1 C, $\left.\mathrm{PhCH}_{2}\right), 51.4$ (3 C, piperidyl-2-yl, piperidyl-6-yl, piperidyl-4-yl), 109.1 (1 C, C-5), 126.6 (2 C, Ph), 126.8 (2 C, Ph), 127.4 (1 C, Ph), 128.9 (2 C, Ph), 129.3 (2 C, Ph), 137.5 (3 C, C-6, Ph), 139.9 (1 C, Ph), 150.8 (1 C, C-2), 163.7 (1 C, C-4), C ( $\left.\mathrm{CH}_{2} \mathrm{~N}\right)$ could not be observed. HRMS (ESI): $m / z[\mathrm{M}+\mathrm{H}]^{+}$Calcd. for $\left[\mathrm{C}_{24} \mathrm{H}_{27} \mathrm{~N}_{3} \mathrm{O}_{2}+\mathrm{H}\right]^{+} 390.2176$, found 390.2186 .

5-Methyl-1-(1-(2-phenoxyphenethyl)piperidin-4-yl)pyrimidine-2,4(1H,3H)-dione (21c). Following the general procedure $\mathrm{B}, \mathbf{1 2 a}(64 \mathrm{mg}, 0.30 \mathrm{mmol}), 28(0.10 \mathrm{~g}, 0.30 \mathrm{mmol})$ and sodium triacetoxyborohydride $(0.13 \mathrm{~g}$, $0.61 \mathrm{mmol})$ in dichloroethane $(10 \mathrm{~mL})$ afforded the BOM-protected intermediate, which was deprotected with TFA $(5.0 \mathrm{~mL})$ in the presence of triethylsilane $(5.0 \mathrm{~mL})$ at $73{ }^{\circ} \mathrm{C}$ for $4 \mathrm{~h}$ to give the $21 \mathrm{c}$ (eluent system: $5 \% \mathrm{MeOH}$ in DCM, $40 \mathrm{mg}, 0.098 \mathrm{mmol}, 32 \%$ yield). ${ }^{1} \mathrm{H}$ NMR (300 MHz, DMSO-d 6 ) $\delta$ ppm 1.52-1.62 
(m, $2 \mathrm{H}$, piperdyl-3a-yl, piperidyl-5a-yl), $1.68-1.84\left(\mathrm{~m}, 5 \mathrm{H}, 5-\mathrm{CH}_{3}\right.$, piperdyl-3b-yl, piperidyl-5b-yl), 1.92-2.04 (m, $2 \mathrm{H}$, piperidyl-2a-yl, piperidyl-6a-yl), 2.50-2.55 (m, $\left.2 \mathrm{H}, \mathrm{CH}_{2} \mathrm{~N}\right), 2.64-2.75\left(\mathrm{~m}, 2 \mathrm{H}, \mathrm{PhCH}_{2}\right)$, $2.92(\mathrm{~d}, J=11.1 \mathrm{~Hz}, 2 \mathrm{H}$, piperidyl-2b-yl, piperidyl-6b-yl), 4.12-4.26 (m, $1 \mathrm{H}$, piperidyl-4-yl), 6.85-6.92 $(\mathrm{m}, 3 \mathrm{H}, \mathrm{Ph}), 7.02-7.15(\mathrm{~m}, 2 \mathrm{H}, \mathrm{Ph}), 7.17-7.26(\mathrm{~m}, 1 \mathrm{H}, \mathrm{Ph}), 7.29-7.38(\mathrm{~m}, 3 \mathrm{H}, \mathrm{Ph}), 7.57(\mathrm{~d}, J=1.2 \mathrm{~Hz}, 1 \mathrm{H}$, $\mathrm{H}-6), 11.16(\mathrm{~s}, 1 \mathrm{H}, \mathrm{NH}) .{ }^{13} \mathrm{C}$ NMR $\left(75 \mathrm{MHz}, \mathrm{DMSO}-\mathrm{d}_{6}\right) \delta \mathrm{ppm} 12.0\left(1 \mathrm{C}, 5-\mathrm{CH}_{3}\right), 27.3\left(1 \mathrm{C} \mathrm{PhCH}_{2}\right), 29.9$ (2 C, piperdyl-3-yl, piperidyl-5-yl), 52.3 (2 C, piperidyl-2-yl, piperidyl-6-yl), 52.3 (1 C, piperidyl-4-yl), 57.9 (1 C, $\left.\mathrm{CH}_{2} \mathrm{~N}\right), 108.9$ (1 C, C-5), 117.1 (2 C, Ph), 119.8 (1 C, Ph), 122.6 (1 C, Ph), 124.2 (1 C, Ph), 127.7 (1 C, Ph), 129.9 (2 C, Ph), 131.1 (1 C, Ph), 131.8 (1 C, Ph), 137.6 (1 C, C-6), 150.8 (1 C, C-2), 153.8 (1 C, Ph), 157.6 (1 C, Ph), 163.6 (1 C, C-4). HRMS (ESI): $m / z[\mathrm{M}+\mathrm{H}]^{+}$Calcd. for $\left[\mathrm{C}_{24} \mathrm{H}_{27} \mathrm{~N}_{3} \mathrm{O}_{3}+\mathrm{H}\right]^{+} 406.2125$, found 406.2133 .

5-Methyl-1-(1-(4-phenoxyphenethyl)piperidin-4-yl)pyrimidine-2,4(1H,3H)-dione (21e). Following the general procedure $B, 12 \mathrm{c}(64 \mathrm{mg}, 0.30 \mathrm{mmol}), 28(0.10 \mathrm{~g}, 0.30 \mathrm{mmol})$ and sodium triacetoxyborohydride $(0.13 \mathrm{~g}$, $0.61 \mathrm{mmol})$ in dichloroethane $(10 \mathrm{~mL})$ afforded the BOM-protected intermediate, which was deprotected with TFA $(5.0 \mathrm{~mL})$ in the presence of triethylsilane $(5.0 \mathrm{~mL})$ at $73^{\circ} \mathrm{C}$ for $4 \mathrm{~h}$ to give the $21 \mathrm{e}$ (eluent system: $5 \% \mathrm{MeOH}$ in DCM, $65 \mathrm{mg}, 0.16 \mathrm{mmol}, 53 \%$ yield). ${ }^{1} \mathrm{H}$ NMR (300 MHz, DMSO-d 6 ) $\delta$ ppm 1.57-1.93 (m, $7 \mathrm{H}, 5-\mathrm{CH}_{3}$, piperdyl-3-yl, piperidyl-5-yl), 1.97-2.16 (m, $2 \mathrm{H}$, piperidyl-2a-yl, piperidyl-6a-yl), $2.54\left(\mathrm{~d}, J=7.3 \mathrm{~Hz}, 2 \mathrm{H}, \mathrm{CH}_{2} \mathrm{~N}\right), 2.64-2.77\left(\mathrm{~m}, 2 \mathrm{H}, \mathrm{PhCH}_{2}\right), 3.04(\mathrm{~d}, J=11.1 \mathrm{~Hz}, 2 \mathrm{H}$, piperidyl-2b-yl, piperidyl-6b-yl), 4.19-4.29 (m, $1 \mathrm{H}$, piperidyl-4-yl), 6.87-6.98 (m, $4 \mathrm{H}, \mathrm{Ph}), 7.04-7.13(\mathrm{~m}, 1 \mathrm{H}, \mathrm{Ph})$, 7.18-7.26 (m, $2 \mathrm{H}, \mathrm{Ph}), 7.29-7.41$ (m, $2 \mathrm{H}, \mathrm{Ph}), 7.60$ (d, J = 0.9 Hz, $1 \mathrm{H}, \mathrm{H}-6), 11.17(\mathrm{~s}, 1 \mathrm{H}, \mathrm{NH}) .{ }^{13} \mathrm{C}$ NMR (75 MHz, DMSO-d 6 ) $\delta$ ppm 12.0 (1 C, 5- $\mathrm{CH}_{3}$ ), 29.9 (2 C, piperdyl-3-yl, piperidyl-5-yl), 32.2 (1 C, $\left.\mathrm{PhCH}_{2}\right), 52.4$ (2 C, piperidyl-2-yl, piperidyl-6-yl), 52.6 (1 C, piperidyl-4-yl), $59.3\left(1 \mathrm{C} \mathrm{CH}_{2} \mathrm{~N}\right), 108.9$ (1 C, C-5), 118.2 (2 C, Ph), 118.7 (2 C, Ph), 123.1 (1 C, Ph), 130.0 (2 C, Ph), 130.1 (2 C, Ph), 135.6 (1 C, Ph), 137.7 (1 C, C-6), 150.8 (1 C, C-2), 154.6 (1 C, Ph), 157.0 (1 C, Ph), 163.7 (1 C, C-4). HRMS (ESI): $\mathrm{m} / z$ [M + $\mathrm{H}]^{+}$Calcd. for $\left[\mathrm{C}_{24} \mathrm{H}_{27} \mathrm{~N}_{3} \mathrm{O}_{3}+\mathrm{H}\right]^{+} 406.2125$, found 406.2124.

5-Methyl-1-(1-(3-(p-tolyloxy)phenethyl)piperidin-4-yl)pyrimidine-2,4(1H,3H)-dione (21f). Following the general procedure $B, \mathbf{1 2 d}(69 \mathrm{mg}, 0.30 \mathrm{mmol}), 28(0.10 \mathrm{~g}, 0.30 \mathrm{mmol})$ and sodium triacetoxyborohydride $(0.13 \mathrm{~g}, 0.61 \mathrm{mmol})$ in dichloroethane $(10 \mathrm{~mL})$ afforded the BOM-protected intermediate, which was deprotected with TFA $(5.0 \mathrm{~mL})$ in the presence of triethylsilane $(5.0 \mathrm{~mL})$ at $73{ }^{\circ} \mathrm{C}$ for $4 \mathrm{~h}$ to give the $\mathbf{2 1 f}$ (eluent system: $5 \% \mathrm{MeOH}$ in DCM, $40 \mathrm{mg}, 0.095 \mathrm{mmol}, 31 \%$ yield). ${ }^{1} \mathrm{H}$ NMR (400 MHz, DMSO-d 6 ) $\delta$ ppm $1.64\left(\mathrm{~d}, J=10.6 \mathrm{~Hz}, 2 \mathrm{H}\right.$, piperdyl-3a-yl, piperidyl-5a-yl), 1.73 - $1.87\left(\mathrm{~m}, 5 \mathrm{H}, 5-\mathrm{CH}_{3}\right.$, piperdyl-3b-yl, piperidyl-5b-yl), $2.05\left(\mathrm{t}, J=11.1 \mathrm{~Hz}, 2 \mathrm{H}\right.$, piperidyl-2a-yl, piperidyl-6a-yl), $2.28\left(\mathrm{~s}, 3 \mathrm{H}, \mathrm{PhCH}_{3}\right), 2.54$ $\left(\mathrm{d}, J=8.4 \mathrm{~Hz}, 2 \mathrm{H}, \mathrm{CH}_{2} \mathrm{~N}\right), 2.72\left(\mathrm{t}, J=7.5 \mathrm{~Hz}, 2 \mathrm{H}, \mathrm{PhCH}_{2}\right), 3.02(\mathrm{~d}, J=11.5 \mathrm{~Hz}, 2 \mathrm{H}$, piperidyl-2b-yl, piperidyl-6b-yl), 4.19-4.29 (m, $1 \mathrm{H}$, piperidyl-4-yl), 6.77 (d, J = 8.1 Hz, $1 \mathrm{H}, \mathrm{Ph}), 6.87(\mathrm{~s}, 1 \mathrm{H}, \mathrm{Ph}), 6.90$ (d, $J=8.3 \mathrm{~Hz}, 2 \mathrm{H}, \mathrm{Ph}), 6.98(\mathrm{~d}, J=7.6 \mathrm{~Hz}, 1 \mathrm{H}, \mathrm{Ph}), 7.19(\mathrm{~d}, J=8.4 \mathrm{~Hz}, 2 \mathrm{H}, \mathrm{Ph}), 7.26(\mathrm{t}, J=7.8 \mathrm{~Hz}, 1 \mathrm{H}$, $\mathrm{Ph}), 7.61$ (s, $1 \mathrm{H}, \mathrm{H}-6), 11.20$ (s, $1 \mathrm{H}, \mathrm{NH}) .{ }^{13} \mathrm{C}$ NMR (101 MHz, DMSO-d 6 ) $\delta$ ppm $12.0\left(1 \mathrm{C}, 5-\mathrm{CH}_{3}\right)$, $20.2\left(1 \mathrm{C}, \mathrm{PhCH}_{3}\right), 30.0$ (2 C, piperdyl-3-yl, piperidyl-5-yl), 32.8 (1 C, $\left.\mathrm{PhCH}_{2}\right), 52.4$ (2 C, piperidyl-2-yl, piperidyl-6-yl), 52.5 (1 C, piperidyl-4-yl), $59.0\left(1 \mathrm{C}, \mathrm{CH}_{2} \mathrm{~N}\right), 108.9$ (1 C, C-5), 115.5 (1 C, Ph), 118.4 (1 C, Ph), 118.8 (2 C, Ph), 123.4 (1 C, Ph), 129.7 (1 C, Ph), 130.3 (2 C, Ph), 132.5 (1 C, Ph), 137.7 (1 C, C-6), 142.7 (1 C, Ph), 150.8 (1 C, C-2), 154.2 (1 C, Ph), 157.1 (1 C, Ph), 163.7 (1 C, C-4). HRMS (ESI): $m / z ~[M+~ H]^{+}$ Calcd. for $\left[\mathrm{C}_{25} \mathrm{H}_{29} \mathrm{~N}_{3} \mathrm{O}_{3}+\mathrm{H}\right]^{+} 420.2282$, found 420.2286 .

1-(1-(3-(3,4-Dichlorophenoxy)phenethyl)piperidin-4-yl)-5-methylpyrimidine-2,4(1H,3H)-dione

(21g). Following the general procedure B, 12e $(85 \mathrm{mg}, 0.30 \mathrm{mmol}), 28(0.10 \mathrm{~g}, 0.30 \mathrm{mmol})$ and sodium triacetoxyborohydride $(0.13 \mathrm{~g}, 0.61 \mathrm{mmol})$ in dichloroethane $(10 \mathrm{~mL})$ afforded the BOM-protected intermediate, which was deprotected with TFA $(5.0 \mathrm{~mL})$ in the presence of triethylsilane $(5.0 \mathrm{~mL})$ at $73{ }^{\circ} \mathrm{C}$ for $4 \mathrm{~h}$ to give the $\mathbf{2 1 g}$ (eluent system: $5 \% \mathrm{MeOH}$ in DCM, $56 \mathrm{mg}, 0.12 \mathrm{mmol}, 39 \%$ yield). ${ }^{1} \mathrm{H}$ NMR $\left(400 \mathrm{MHz}, \mathrm{DMSO}-\mathrm{d}_{6}\right) \delta$ ppm $1.79\left(\mathrm{~s}, 3 \mathrm{H}, 5-\mathrm{CH}_{3}\right), 1.91-2.06(\mathrm{~m}, 2 \mathrm{H}$, piperdyl-3a-yl, piperidyl-5a-yl), 2.10-2.19 (m, $2 \mathrm{H}$, piperdyl-3b-yl, piperidyl-5b-yl), 2.96-3.08 (m, $\left.2 \mathrm{H}, \mathrm{PhCH}_{2}\right), 3.09-3.22(\mathrm{~m}, 2 \mathrm{H}$, piperidyl-2a-yl, piperidyl-6a-yl), 3.25-3.37 (m, $\left.2 \mathrm{H}, \mathrm{CH}_{2} \mathrm{~N}\right), 3.56-3.73(\mathrm{~m}, 2 \mathrm{H}$, piperidyl-2b-yl, piperidyl-6b-yl), 4.46-4.59 (m, $1 \mathrm{H}$, piperidyl-4-yl), 7.02 (dd, J = 8.9, $2.9 \mathrm{~Hz}, 2 \mathrm{H}, \mathrm{Ph}$ ), 7.06 (br. s., 1 
$\mathrm{H}, \mathrm{Ph}), 7.15(\mathrm{~d}, J=7.3 \mathrm{~Hz}, 1 \mathrm{H}, \mathrm{Ph}), 7.30(\mathrm{~d}, J=2.8 \mathrm{~Hz}, 1 \mathrm{H}, \mathrm{Ph}), 7.34-7.46$ (m, $2 \mathrm{H}, \mathrm{H}-6, \mathrm{Ph}), 7.65$ (d, $J=8.9 \mathrm{~Hz}, 1 \mathrm{H}, \mathrm{Ph}), 11.34(\mathrm{~s}, 1 \mathrm{H}, \mathrm{NH}) .{ }^{13} \mathrm{C}$ NMR $\left(101 \mathrm{MHz}, \mathrm{DMSO}-\mathrm{d}_{6}\right) \delta \mathrm{ppm} 12.2\left(1 \mathrm{C}, 5-\mathrm{CH}_{3}\right), 27.1(2$ C, piperdyl-3-yl, piperidyl-5-yl), 29.4 (1 C, $\left.\mathrm{PhCH}_{2}\right), 50.5$ (1 C, piperidyl-4-yl), 51.2 (2 C, piperidyl-2-yl, piperidyl-6-yl), 56.3 ( $\left.1 \mathrm{C}, \mathrm{CH}_{2} \mathrm{~N}\right), 109.3$ (1 C, C-5), 117.8 (1 C, Ph), 118.7 (1 C, Ph), 119.5 (1 C, Ph), 120.2 (1 C, Ph), 125.16 (d, J = 43.5 Hz, 1 C, Ph), 130.6 (1 C, Ph), 131.6 (2 C, Ph), 132.0 (1 C, Ph), 137.4 (1 C, C-6), 139.6 (1 C, Ph), 150.7 (1 C, C-2), 155.8 (1 C, Ph), 156.4 (1 C, Ph), 163.7 (1 C, C-4). HRMS (ESI): m/z [M + $\mathrm{H}]^{+}$Calcd. for $\left[\mathrm{C}_{24} \mathrm{H}_{25} \mathrm{Cl}_{2} \mathrm{~N}_{3} \mathrm{O}_{3}+\mathrm{H}\right]^{+} 474.1346$, found 474.1333 .

1-(1-(3-(4-Chlorophenoxy)phenethyl)piperidin-4-yl)-5-methylpyrimidine-2,4(1H,3H)-dione (21h). Following the general procedure B, 12f $(75 \mathrm{mg}, 0.30 \mathrm{mmol}), 28(0.10 \mathrm{~g}, 0.30 \mathrm{mmol})$ and sodium triacetoxyborohydride $(0.13 \mathrm{~g}, 0.61 \mathrm{mmol})$ in dichloroethane $(10 \mathrm{~mL})$ afforded the BOM-protected intermediate, which was deprotected with TFA $(5.0 \mathrm{~mL})$ in the presence of triethylsilane $(5.0 \mathrm{~mL})$ at $73{ }^{\circ} \mathrm{C}$ for $4 \mathrm{~h}$ to give the $21 \mathrm{~h}$ (eluent system: $5 \% \mathrm{MeOH}$ in DCM, $40 \mathrm{mg}, 0.091 \mathrm{mmol}, 30 \%$ yield). ${ }^{1} \mathrm{H}$ NMR (300 MHz, DMSO-d 6 ) $\delta$ ppm 1.57-1.67 (m, 2 H, piperdyl-3a-yl, piperidyl-5a-yl), 1.68-1.87 (m, 5 $\mathrm{H}, 5-\mathrm{CH}_{3}$, piperdyl-3b-yl, piperidyl-5b-yl), $2.03(\mathrm{t}, J=12.3 \mathrm{~Hz}, 2 \mathrm{H}$, piperidyl-2a-yl, piperidyl-6a-yl), $2.54\left(\mathrm{~d}, J=8.5 \mathrm{~Hz}, 2 \mathrm{H}, \mathrm{CH}_{2} \mathrm{~N}\right), 2.72\left(\mathrm{t}, J=7.4 \mathrm{~Hz}, 2 \mathrm{H}, \mathrm{PhCH}_{2}\right), 3.00(\mathrm{~d}, J=12.0 \mathrm{~Hz}, 2 \mathrm{H}$, piperidyl-2b-yl, piperidyl-6b-yl), $4.22(\mathrm{tt}, J=12.1,4.5 \mathrm{~Hz}, 1 \mathrm{H}$, piperidyl-4-yl), $6.83(\mathrm{dd}, J=7.8,1.9 \mathrm{~Hz}, 1 \mathrm{H}, \mathrm{Ph}), 6.92$ $(\mathrm{s}, 1 \mathrm{H}, \mathrm{Ph}), 6.96-7.06(\mathrm{~m}, 3 \mathrm{H}, \mathrm{Ph}), 7.24-7.33(\mathrm{~m}, 1 \mathrm{H}, \mathrm{Ph}), 7.38-7.43(\mathrm{~m}, 2 \mathrm{H}, \mathrm{Ph}), 7.59(\mathrm{~s}, 1 \mathrm{H}, \mathrm{H}-6)$, $11.17(\mathrm{~s}, 1 \mathrm{H}, \mathrm{NH}) .{ }^{13} \mathrm{C}$ NMR $\left(75 \mathrm{MHz}, \mathrm{DMSO}-\mathrm{d}_{6}\right) \delta \mathrm{ppm} 12.0\left(1 \mathrm{C}, 5-\mathrm{CH}_{3}\right), 30.0$ (2 C, piperdyl-3-yl, piperidyl-5-yl), 32.7 (1 C, $\left.\mathrm{PhCH}_{2}\right), 52.4$ (2 C, piperidyl-2-yl, piperidyl-6-yl), 52.5 (1 C, piperidyl-4-yl), 59.0 (1 C, $\left.\mathrm{CH}_{2} \mathrm{~N}\right), 108.9$ (1 C, C-5), 116.3 (1 C, Ph), 119.2 (1 C, Ph), 120.0 (2 C, Ph), 124.3 (1 C, Ph), 126.9 (1 C, Ph), 129.8 (2 C, Ph), 129.9 (1 C, Ph), 137.6 (1 C, C-6), 143.0 (1 C, Ph), 150.8 (1 C, C-2), 155.8 (1 C, Ph), 156.1 (1 C, Ph), 163.7 (1 C, C-4). HRMS (ESI): $m / z[\mathrm{M}+\mathrm{H}]^{+}$Calcd. for $\left[\mathrm{C}_{24} \mathrm{H}_{26} \mathrm{ClN}_{3} \mathrm{O}_{3}+\mathrm{H}\right]^{+} 440.1736$, found 440.1750 .

1-(1-(3-(4-Methoxyphenoxy)phenethyl)piperidin-4-yl)-5-methylpyrimidine-2,4(1H,3H)-dione (21i). Following the general procedure $\mathrm{B}, \mathbf{1 2 \mathrm { g }}(74 \mathrm{mg}, 0.30 \mathrm{mmol}), 28(0.10 \mathrm{~g}, 0.30 \mathrm{mmol})$ and sodium triacetoxyborohydride $(0.13 \mathrm{~g}, 0.61 \mathrm{mmol})$ in dichloroethane $(10 \mathrm{~mL})$ afforded the BOM-protected intermediate, which was deprotected with TFA $(5.0 \mathrm{~mL})$ in the presence of triethylsilane $(5.0 \mathrm{~mL})$ at $73^{\circ} \mathrm{C}$ for $4 \mathrm{~h}$ to give the $21 \mathrm{i}$ (eluent system: $5 \% \mathrm{MeOH}$ in DCM, $40 \mathrm{mg}, 0.092 \mathrm{mmol}, 30 \%$ yield). ${ }^{1} \mathrm{H}$ NMR $\left(400 \mathrm{MHz}, \mathrm{DMSO}-\mathrm{d}_{6}\right) \delta$ ppm 1.65 (d, J = $10.6 \mathrm{~Hz}, 2 \mathrm{H}$, piperdyl-3a-yl, piperidyl-5a-yl), 1.74-1.87 (m, 5 $\mathrm{H}$, piperdyl-3b-yl, piperidyl-5b-yl, 5- $\left.\mathrm{CH}_{3}\right), 2.05(\mathrm{t}, J=11.3 \mathrm{~Hz}, 2 \mathrm{H}$, piperidyl-2a-yl, piperidyl-6a-yl), 2.52-2.56 (m, $\left.2 \mathrm{H}, \mathrm{CH}_{2} \mathrm{~N}\right), 2.70\left(\mathrm{t}, J=8.3 \mathrm{~Hz}, 2 \mathrm{H}, \mathrm{PhCH}_{2}\right), 3.02(\mathrm{~d}, J=11.4 \mathrm{~Hz}, 2 \mathrm{H}$, piperidyl-2b-yl, piperidyl-6b-yl), $3.74\left(\mathrm{~s}, 3 \mathrm{H}, \mathrm{OCH}_{3}\right), 4.19-4.29(\mathrm{~m}, 1 \mathrm{H}$, piperidyl-4-yl), $6.72(\mathrm{~d}, J=8.1 \mathrm{~Hz}, 1 \mathrm{H}, \mathrm{Ph})$, 6.83 (s, 1 H, Ph), 6.92-7.01 (m, 5 H, Ph), 7.24 (t, $J=7.9$ Hz, 1 H, Ph), 7.61 (s, 1 H, H-6), 11.20 (s, 1 H, NH). ${ }^{13} \mathrm{C}$ NMR (101 MHz, DMSO-d 6 ) $\delta$ ppm $12.0\left(1 \mathrm{C}, 5-\mathrm{CH}_{3}\right), 30.0$ (2 C, piperdyl-3-yl, piperidyl-5-yl), 32.8 (1 C, $\left.\mathrm{PhCH}_{2}\right), 52.4$ (2 C, piperidyl-2-yl, piperidyl-6-yl), 52.5 (1 C, piperidyl-4-yl), $55.4\left(1 \mathrm{C}, \mathrm{OCH}_{3}\right), 59.0$ (1 C, $\left.\mathrm{CH}_{2} \mathrm{~N}\right), 108.9$ (1 C, C-5), 114.7 (1 C, Ph), 115.0 (2 C, Ph), 117.6 (1 C, Ph), 120.6 (2 C, Ph), 123.0 (1 C, Ph), 129.6 (1 C, Ph), 137.7 (1 C, C-6), 142.6 (1 C, Ph), 149.4 (1 C, Ph), 150.8 (1 C, C-2), 155.5 (1 C, Ph), 157.9 (1 C, Ph), 163.7 (1 C, C-4). HRMS (ESI): $m / z[\mathrm{M}+\mathrm{H}]^{+}$Calcd. for $\left[\mathrm{C}_{25} \mathrm{H}_{29} \mathrm{~N}_{3} \mathrm{O}_{4}+\mathrm{H}\right]^{+} 436.2231$, found 436.2234 .

1-(1-(3-(3-Chlorophenoxy)phenethyl)piperidin-4-yl)-5-methylpyrimidine-2,4(1H,3H)-dione (21j). Following the general procedure $\mathrm{B}, \mathbf{1 2} \mathrm{h}(75 \mathrm{mg}, 0.30 \mathrm{mmol}), 28(0.10 \mathrm{~g}, 0.30 \mathrm{mmol})$ and sodium triacetoxyborohydride $(0.13 \mathrm{~g}, 0.61 \mathrm{mmol})$ in dichloroethane $(10 \mathrm{~mL})$ afforded the BOM-protected intermediate, which was deprotected with TFA $(5.0 \mathrm{~mL})$ in the presence of triethylsilane $(5.0 \mathrm{~mL})$ at $73{ }^{\circ} \mathrm{C}$ for $4 \mathrm{~h}$ to give the $21 \mathrm{j}$ (eluent system: $5 \% \mathrm{MeOH}$ in DCM, $63 \mathrm{mg}, 0.14 \mathrm{mmol}, 47 \%$ yield). ${ }^{1} \mathrm{H}$ NMR $\left(400 \mathrm{MHz}, \mathrm{DMSO}-\mathrm{d}_{6}\right) \delta \mathrm{ppm} 1.65(\mathrm{~d}, J=9.8 \mathrm{~Hz}, 2 \mathrm{H}$, piperdyl-3a-yl, piperidyl-5a-yl), 1.71-1.89 (m, $5 \mathrm{H}$, piperdyl-3b-yl, piperidyl-5b-yl, 5- $\left.\mathrm{CH}_{3}\right), 2.06(\mathrm{t}, J=11.0 \mathrm{~Hz}, 2 \mathrm{H}$, piperidyl-2a-yl, piperidyl-6a-yl), 2.53-2.62 (m, $\left.2 \mathrm{H}, \mathrm{CH}_{2} \mathrm{~N}\right), 2.69-2.79\left(\mathrm{~m}, 2 \mathrm{H}, \mathrm{PhCH}_{2}\right), 3.03(\mathrm{~d}, J=11.6 \mathrm{~Hz}, 2 \mathrm{H}$, piperidyl-2b-yl, piperidyl-6b-yl), 4.17-4.31 (m, $1 \mathrm{H}$, piperidyl-4-yl), $6.88(\mathrm{dd}, J=8.1,1.8 \mathrm{~Hz}, 1 \mathrm{H}, \mathrm{Ph}), 6.92-7.00(\mathrm{~m}, 2 \mathrm{H}$, $\mathrm{Ph}), 7.02(\mathrm{t}, J=2.2 \mathrm{~Hz}, 1 \mathrm{H}, \mathrm{Ph}), 7.08(\mathrm{~d}, J=7.8 \mathrm{~Hz}, 1 \mathrm{H}, \mathrm{Ph}), 7.15-7.21(\mathrm{~m}, 1 \mathrm{H}, \mathrm{Ph}), 7.33(\mathrm{t}, J=7.8 \mathrm{~Hz}, 1$ 
$\mathrm{H}, \mathrm{Ph}), 7.37-7.43(\mathrm{~m}, 1 \mathrm{H}, \mathrm{Ph}), 7.60(\mathrm{~d}, J=0.9 \mathrm{~Hz}, 1 \mathrm{H}, \mathrm{H}-6), 11.20(\mathrm{~s}, 1 \mathrm{H}, \mathrm{NH}) .{ }^{13} \mathrm{C}$ NMR $(101 \mathrm{MHz}$, DMSO-d 6 ) $\delta$ ppm 12.0 (1 C, 5- $\mathrm{CH}_{3}$ ), 30.0 (2 C, piperdyl-3-yl, piperidyl-5-yl), $32.7\left(1 \mathrm{C}, \mathrm{PhCH}_{2}\right), 52.4$

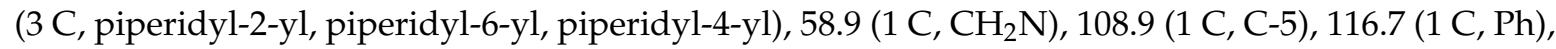
116.8 (1 C, Ph), 118.0 (1 C, Ph), 119.6 (1 C, Ph), 123.0 (1 C, Ph), 124.7 (1 C, Ph), 130.0 (1 C, Ph), 131.4 (1 C, Ph), 133.9 (1 C, Ph), 137.6 (1 C, C-6), 143.1 (1 C, Ph), 150.8 (1 C, C-2), 155.5 (1 C, Ph), 158.1 (1 C, Ph), 163.7 (1 C, C-4). HRMS (ESI): $\mathrm{m} / \mathrm{z}[\mathrm{M}+\mathrm{H}]^{+}$Calcd. for $\left[\mathrm{C}_{24} \mathrm{H}_{26} \mathrm{ClN}_{3} \mathrm{O}_{3}+\mathrm{H}\right]^{+} 440.1736$, found 440.1740 .

4-(3-(2-(4-(5-Methyl-2,4-dioxo-3,4-dihydropyrimidin-1(2H)-yl)piperidin-1-yl)ethyl)phenoxy)benzonitrile (21k). Following the general procedure B, 18a $(72 \mathrm{mg}, 0.30 \mathrm{mmol}), 28(0.10 \mathrm{~g}, 0.30 \mathrm{mmol})$ and sodium triacetoxyborohydride $(0.13 \mathrm{~g}, 0.61 \mathrm{mmol})$ in dichloroethane $(10 \mathrm{~mL})$ afforded the BOM-protected intermediate, which was deprotected with TFA $(5.0 \mathrm{~mL})$ in the presence of triethylsilane $(5.0 \mathrm{~mL})$ at $73{ }^{\circ} \mathrm{C}$ for $4 \mathrm{~h}$ to give the $21 \mathrm{k}$ (eluent system: $5 \% \mathrm{MeOH}$ in DCM, $50 \mathrm{mg}, 0.12 \mathrm{mmol}, 43 \%$ yield). ${ }^{1} \mathrm{H}$ NMR $\left(400 \mathrm{MHz}, \mathrm{DMSO}-\mathrm{d}_{6}\right) \delta \mathrm{ppm} 1.79\left(\mathrm{~s}, 3 \mathrm{H}, 5-\mathrm{CH}_{3}\right), 2.00(\mathrm{~d}, J=10.8 \mathrm{~Hz}, 2 \mathrm{H}$, piperdyl-3a-yl, piperidyl-5a-yl), 2.21 (d, $J=11.6 \mathrm{~Hz}, 2 \mathrm{H}$, piperdyl-3b-yl, piperidyl-5b-yl), 3.05 (br. s., $2 \mathrm{H}, \mathrm{PhCH}_{2}$ ), 3.15 (br. s., 2 H, piperidyl-2a-yl, piperidyl-6a-yl), 3.32 (s, $2 \mathrm{H}, \mathrm{CH}_{2} \mathrm{~N}$ ), 3.66 (br. s., $2 \mathrm{H}$, piperidyl-2b-yl, piperidyl-6b-yl), 4.44-4.63 (m, $1 \mathrm{H}$, piperidyl-4-yl), 7.03-7.16 (m, $4 \mathrm{H}, \mathrm{Ph}), 7.21(\mathrm{~d}, J=6.6 \mathrm{~Hz}, 1 \mathrm{H}, \mathrm{Ph})$, 7.35-7.53 (m, 2 H, Ph, H-6), 7.86 (d, J = 8.4 Hz, 2 H, Ph), 11.32 (br. s., $1 \mathrm{H}, \mathrm{NH}) .{ }^{13} \mathrm{C} \mathrm{NMR}(101 \mathrm{MHz}$, DMSO-d 6 ) $\delta$ ppm $12.2\left(1 \mathrm{C}, 5-\mathrm{CH}_{3}\right), 27.1$ (2 C, piperdyl-3-yl, piperidyl-5-yl), $29.3\left(1 \mathrm{C}, \mathrm{PhCH}_{2}\right), 50.3(1$ C, piperidyl-4-yl), $51.2\left(2 \mathrm{C}\right.$, piperidyl-2-yl, piperidyl-6-yl), $56.2\left(1 \mathrm{C}, \mathrm{CH}_{2} \mathrm{~N}\right), 105.2(1 \mathrm{C}, \mathrm{Ph}), 109.3(1 \mathrm{C}$, C-5), 118.1 (2 C, Ph), 118.7 (2 C, Ph, CN), 120.5 (1 C, Ph), 125.6 (1 C, Ph), 130.7 (1 C, Ph), 134.7 (2 C, Ph), 137.3 (1 C, C-6), 139.7 (1 C, Ph), 150.7 (1 C, C-2), 154.7 (1 C, Ph), 161.0 (1 C, Ph), 163.6 (1 C. C-4). HRMS (ESI): $m / z[\mathrm{M}+\mathrm{H}]^{+}$Calcd. for $\left[\mathrm{C}_{25} \mathrm{H}_{26} \mathrm{~N}_{4} \mathrm{O}_{3}+\mathrm{H}\right]^{+} 431.2078$, found 431.2085 .

5-Methyl-1-(1-(3-(4-nitrophenoxy)phenethyl)piperidin-4-yl)pyrimidine-2,4(1H,3H)-dione (211). Following the general procedure $\mathrm{B}, \mathbf{1 8 b}(78 \mathrm{mg}, 0.30 \mathrm{mmol}), 28(0.10 \mathrm{~g}, 0.30 \mathrm{mmol})$ and sodium triacetoxyborohydride $(0.13 \mathrm{~g}, 0.61 \mathrm{mmol})$ in dichloroethane $(10 \mathrm{~mL})$ afforded the BOM-protected intermediate, which was deprotected with TFA $(5.0 \mathrm{~mL})$ in the presence of triethylsilane $(5.0 \mathrm{~mL})$ at $73^{\circ} \mathrm{C}$ for $4 \mathrm{~h}$ to give the 211 (eluent system: $5 \% \mathrm{MeOH}$ in DCM, $67 \mathrm{mg}, 0.15 \mathrm{mmol}, 40 \%$ yield). ${ }^{1} \mathrm{H}$ NMR (400 MHz, DMSO-d 6 ) $\delta$ ppm 1.79 (s, $\left.3 \mathrm{H}, 5-\mathrm{CH}_{3}\right), 1.93-2.06(\mathrm{~m}, 2 \mathrm{H}$, piperdyl-3a-yl, piperidyl-5a-yl), 2.20 (d, J = 11.6 Hz, $2 \mathrm{H}$, piperdyl-3b-yl, piperidyl-5b-yl), 2.98-3.08 (m, $\left.2 \mathrm{H}, \mathrm{PhCH}_{2}\right), 3.09-3.24(\mathrm{~m}, 2 \mathrm{H}$, piperidyl-2a-yl, piperidyl-6a-yl), 3.41-3.55 (m, $\left.2 \mathrm{H}, \mathrm{CH}_{2} \mathrm{~N}\right), 3.67(\mathrm{~d}, J=10.6 \mathrm{~Hz}, 2 \mathrm{H}$, piperidyl-2b-yl, piperidyl-6b-yl), 4.44-4.62 (m, $1 \mathrm{H}$, piperidyl-4-yl), 7.09-7.17 (m, $4 \mathrm{H}, \mathrm{Ph}), 7.24(\mathrm{~d}, J=7.5 \mathrm{~Hz}, 1 \mathrm{H}, \mathrm{Ph}), 7.38(\mathrm{~s}, 1 \mathrm{H}$, $\mathrm{H}-6), 7.49(\mathrm{t}, J=7.8 \mathrm{~Hz}, 1 \mathrm{H}, \mathrm{Ph}), 8.27(\mathrm{~d}, J=9.1 \mathrm{~Hz}, 2 \mathrm{H}, \mathrm{Ph}), 11.32(\mathrm{~s}, 1 \mathrm{H}, \mathrm{NH}) .{ }^{13} \mathrm{C} \mathrm{NMR}(101 \mathrm{MHz}$, DMSO-d 6 ) $\delta$ ppm $12.2\left(1 \mathrm{C}, 5-\mathrm{CH}_{3}\right), 27.0$ (2 C, piperdyl-3-yl, piperidyl-5-yl), $29.3\left(1 \mathrm{C}, \mathrm{PhCH}_{2}\right), 50.5$ (1 C, piperidyl-4-yl), 51.2 (2 C, piperidyl-2-yl, piperidyl-6-yl), $56.2\left(1 \mathrm{C}, \mathrm{CH}_{2} \mathrm{~N}\right), 109.3(1 \mathrm{C}, \mathrm{C}-5), 117.5$ (2 C, Ph), 118.9 (1 C, Ph), 120.7 (1 C, Ph), 125.9 (1 C, Ph), 126.2 (2 C, Ph), 130.8 (1 C, Ph), 137.3 (1 C, C-6), 139.4 (1 C, Ph), 142.3 (1 C, Ph), 150.7 (1 C, C-2), 154.5 (1 C, Ph), 162.7 (1 C, Ph), 163.6 (1 C, C-4). HRMS (ESI): $m / z[\mathrm{M}+\mathrm{H}]^{+}$Calcd. for $\left[\mathrm{C}_{24} \mathrm{H}_{26} \mathrm{~N}_{4} \mathrm{O}_{5}+\mathrm{H}\right]^{+}$451.1976, found 451.1971.

5-Methyl-1-(1-(3-(4-(trifluoromethyl)phenoxy)phenethyl)piperidin-4-yl)pyrimidine-2,4(1H,3H)-dione (21m). Following the general procedure $B, \mathbf{1 8 c}(85 \mathrm{mg}, 0.30 \mathrm{mmol}), 28(0.10 \mathrm{~g}, 0.30 \mathrm{mmol})$ and sodium triacetoxyborohydride $(0.13 \mathrm{~g}, 0.61 \mathrm{mmol})$ in dichloroethane $(10 \mathrm{~mL})$ afforded the BOM-protected intermediate, which was deprotected with TFA $(5.0 \mathrm{~mL})$ in the presence of triethylsilane $(5.0 \mathrm{~mL})$ at $73{ }^{\circ} \mathrm{C}$ for $4 \mathrm{~h}$ to give the $21 \mathrm{~m}$ (eluent system: $5 \% \mathrm{MeOH}$ in DCM, $64 \mathrm{mg}, 0.14 \mathrm{mmol}, 44 \%$ yield). ${ }^{1} \mathrm{H}$ NMR (400 MHz, DMSO-d 6 ) $\delta$ ppm 1.79 (s, $3 \mathrm{H}, 5-\mathrm{CH}_{3}$ ), 2.00 (d, J = 12.0 Hz, $2 \mathrm{H}$, piperdyl-3a-yl, piperidyl-5a-yl), $2.20\left(\mathrm{~d}, J=11.4 \mathrm{~Hz}, 2 \mathrm{H}\right.$, piperdyl-3b-yl, piperidyl-5b-yl), $2.99-3.08\left(\mathrm{~m}, 2 \mathrm{H}, \mathrm{PhCH}_{2}\right)$, $3.16\left(\mathrm{~d}, J=9.5 \mathrm{~Hz}, 2 \mathrm{H}\right.$, piperidyl-2a-yl, piperidyl-6a-yl), 3.39-3.55 $\left(\mathrm{m}, 2 \mathrm{H}, \mathrm{CH}_{2} \mathrm{~N}\right), 3.67(\mathrm{~d}, J=10.6$ $\mathrm{Hz}, 2 \mathrm{H}$, piperidyl-2b-yl, piperidyl-6b-yl), 4.47-4.59 (m, $1 \mathrm{H}$, piperidyl-4-yl), 7.05 (d, J = 7.9 Hz, $1 \mathrm{H}$, $\mathrm{Ph}), 7.10-7.20$ (m, $4 \mathrm{H}, \mathrm{Ph}), 7.39$ (br. s., $1 \mathrm{H}, \mathrm{H}-6), 7.44$ (t, $J=7.8 \mathrm{~Hz}, 1 \mathrm{H}, \mathrm{Ph}), 7.75$ (d, J = 8.5 Hz, $2 \mathrm{H}$, $\mathrm{Ph}), 11.32$ (s, $1 \mathrm{H}, \mathrm{NH}) .{ }^{19} \mathrm{~F}$ NMR (377 MHz, DMSO-d 6 ) $\delta \mathrm{ppm}-60.16$ (s, 3 F). ${ }^{13} \mathrm{C}$ NMR $(101 \mathrm{MHz}$, DMSO-d 6 ) $\delta$ ppm $12.2\left(1 \mathrm{C}, 5-\mathrm{CH}_{3}\right), 27.1$ (2 C, piperdyl-3-yl, piperidyl-5-yl), $29.3\left(1 \mathrm{C}, \mathrm{PhCH}_{2}\right), 50.4$ (1 C, piperidyl-4-yl), 51.1 (2 C, piperidyl-2-yl, piperidyl-6-yl), $56.3\left(1 \mathrm{C}, \mathrm{CH}_{2} \mathrm{~N}\right), 109.3(1 \mathrm{C}, \mathrm{C}-5), 118.0$ (2 
C, Ph), 118.4 (1 C, Ph), 120.2 (1 C, Ph), 123.20 (q, J = 32.6 Hz, 1 C, Ph), 124.27 (q, J = 271.3 Hz, 1 C, CF $)$, 125.2 (1 C, Ph), 127.5 (2 C, Ph), 130.6 (1 C, Ph), 137.3 (1 C, C-6), 139.6 (1 C, Ph), 150.7 (1 C, C-2), 155.2 (1 C, Ph), $160.3(1 \mathrm{C}, \mathrm{Ph}), 163.6(1 \mathrm{C}, \mathrm{C}-4)$. HRMS (ESI): $\mathrm{m} / \mathrm{z}[\mathrm{M}+\mathrm{H}]^{+}$Calcd. for $\left[\mathrm{C}_{25} \mathrm{H}_{26} \mathrm{~F}_{3} \mathrm{~N}_{3} \mathrm{O}_{3}+\mathrm{H}\right]^{+}$ 474.1999, found 474.1989.

1-(1-(3-(Benzyloxy)phenethyl)piperidin-4-yl)-5-methylpyridine-2,4(1H,3H)-dione (21n). Following the general procedure $B, 15 \mathrm{a}(69 \mathrm{mg}, 0.30 \mathrm{mmol}), 28(0.10 \mathrm{~g}, 0.30 \mathrm{mmol})$ and sodium triacetoxyborohydride $(0.13 \mathrm{~g}, 0.61 \mathrm{mmol})$ in dichloroethane $(10 \mathrm{~mL})$ afforded the BOM-protected intermediate, which was deprotected with TFA $(5.0 \mathrm{~mL})$ in the presence of triethylsilane $(5.0 \mathrm{~mL})$ at $73{ }^{\circ} \mathrm{C}$ for $4 \mathrm{~h}$ to give the $21 \mathrm{n}$ (eluent system: $5 \% \mathrm{MeOH}$ in DCM, $58 \mathrm{mg}, 0.14 \mathrm{mmol}, 46 \%$ yield). ${ }^{1} \mathrm{H}$ NMR (400 MHz, DMSO-d 6 ) $\delta$ ppm $1.66\left(\mathrm{~d}, J=10.8 \mathrm{~Hz}, 2 \mathrm{H}\right.$, piperdyl-3a-yl, piperidyl-5a-yl), 1.74-1.90 (m, $5 \mathrm{H}, 5-\mathrm{CH}_{3}$, piperdyl-3b-yl, piperidyl-5b-yl), $2.07(\mathrm{t}, J=11.3 \mathrm{~Hz}, 2 \mathrm{H}$, piperidyl-2a-yl, piperidyl-6a-yl), $2.55(\mathrm{t}, J=9.0 \mathrm{~Hz}, 2 \mathrm{H}$, $\left.\mathrm{CH}_{2} \mathrm{~N}\right), 2.70\left(\mathrm{t}, J=7.4 \mathrm{~Hz}, 2 \mathrm{H}, \mathrm{PhCH}_{2}\right), 3.04(\mathrm{~d}, J=11.3 \mathrm{~Hz}, 2 \mathrm{H}$, piperidyl-2b-yl, piperidyl-6b-yl), 4.20-4.31 (m, $1 \mathrm{H}$, piperidyl-4-yl), $5.08\left(\mathrm{~s}, 2 \mathrm{H},(\mathrm{Ph}) \mathrm{CH}_{2} \mathrm{O}\right), 6.82(\mathrm{t}, J=7.8 \mathrm{~Hz}, 2 \mathrm{H}, \mathrm{Ph}), 6.90(\mathrm{~s}, 1 \mathrm{H}, \mathrm{Ph})$, $7.19(\mathrm{t}, J=7.9 \mathrm{~Hz}, 1 \mathrm{H}, \mathrm{Ph}), 7.30-7.35(\mathrm{~m}, 1 \mathrm{H}, \mathrm{Ph}), 7.39(\mathrm{t}, J=7.4 \mathrm{~Hz}, 2 \mathrm{H}, \mathrm{Ph}), 7.43-7.48(\mathrm{~m}, 2 \mathrm{H}, \mathrm{Ph})$, 7.63 (s, $1 \mathrm{H}, \mathrm{H}-6), 11.20(\mathrm{~s}, 1 \mathrm{H}, \mathrm{NH}) .{ }^{13} \mathrm{C}$ NMR $\left(101 \mathrm{MHz}, \mathrm{DMSO}-\mathrm{d}_{6}\right) \delta \mathrm{ppm} 12.0\left(1 \mathrm{C}, 5-\mathrm{CH}_{3}\right), 30.0(2 \mathrm{C}$, piperdyl-3-yl, piperidyl-5-yl), $33.0\left(1 \mathrm{C}, \mathrm{PhCH}_{2}\right), 52.4$ (2 C, piperidyl-2-yl, piperidyl-6-yl), $52.4(1 \mathrm{C}$, piperidyl-4-yl), $59.2\left(1 \mathrm{C}, \mathrm{CH}_{2} \mathrm{~N}\right), 69.0\left(1 \mathrm{C},(\mathrm{Ph}) \mathrm{CH}_{2} \mathrm{O}\right), 108.9(1 \mathrm{C}, \mathrm{C}-5), 112.1(1 \mathrm{C}, \mathrm{Ph}), 115.2(1 \mathrm{C}, \mathrm{Ph})$, 121.1 (1 C, Ph), 127.7 (2 C, Ph), 127.8 (1 C, Ph), 128.4 (2 C, Ph), 129.2 (1 C, Ph), 137.2 (1 C, Ph), 137.7 (1 C, C-6), 142.0 (1 C, Ph), 150.8 (1 C, C-2), 158.4 (1 C, Ph), 163.7 (1 C, C-4). HRMS (ESI): $m / z$ [M + H] ${ }^{+}$Calcd. for $\left[\mathrm{C}_{25} \mathrm{H}_{29} \mathrm{~N}_{3} \mathrm{O}_{3}+\mathrm{H}\right]^{+} 420.2282$, found 420.2277 .

1-(1-(3-((2-Chlorobenzyl)oxy)phenethyl)piperidin-4-yl)-5-methylpyrimidine-2,4(1H,3H)-dione (21o). Following the general procedure $\mathrm{B}, \mathbf{1 5 b}(79 \mathrm{mg}, 0.30 \mathrm{mmol}), \mathbf{2 8}(0.10 \mathrm{~g}, 0.30 \mathrm{mmol})$ and sodium triacetoxyborohydride $(0.13 \mathrm{~g}, 0.61 \mathrm{mmol})$ in dichloroethane $(10 \mathrm{~mL})$ afforded the BOM-protected intermediate, which was deprotected with TFA $(5.0 \mathrm{~mL})$ in the presence of triethylsilane $(5.0 \mathrm{~mL})$ at $73{ }^{\circ} \mathrm{C}$ for $4 \mathrm{~h}$ to give the $21 \mathrm{o}$ (eluent system: $5 \% \mathrm{MeOH}$ in DCM, $90 \mathrm{mg}, 0.20 \mathrm{mmol}, 65 \%$ yield). ${ }^{1} \mathrm{H}$ NMR (400 MHz, DMSO-d 6 ) $\delta$ ppm 1.73-2.23 (m, $7 \mathrm{H}$, piperdyl-3-yl, piperidyl-5-yl, 5- $\left.\mathrm{CH}_{3}\right), 2.92$ (br. s., $\left.2 \mathrm{H}, \mathrm{PhCH}_{2}\right), 3.21-3.67(\mathrm{~m}, 4 \mathrm{H}$, piperidyl-2-yl, piperidyl-6-yl), 4.35-4.68 (m, $1 \mathrm{H}$, piperidyl-4-yl), $5.12\left(\mathrm{~s}, 2 \mathrm{H},(\mathrm{Ph}) \mathrm{CH}_{2} \mathrm{O}\right), 6.85-6.93(\mathrm{~m}, 2$ H, Ph), 6.97 (br. s., 1 H, Ph), 7.26 (t, J = 7.8 Hz, 1 H, Ph), 7.36-7.49 (m, 4 H, Ph), 7.52 (s, 1 H, H-6), 11.31 (br. s., $1 \mathrm{H}, \mathrm{NH}), 2 \mathrm{H}\left(\mathrm{CH}_{2} \mathrm{~N}\right)$ could not be observed. ${ }^{13} \mathrm{C}$ NMR $\left(101 \mathrm{MHz}, \mathrm{DMSO}-\mathrm{d}_{6}\right) \delta p p m 12.2(1 \mathrm{C}$, 5- $\mathrm{CH}_{3}$ ), 27.0 (2 C, piperdyl-3-yl, piperidyl-5-yl), 30.2 (1 C, $\left.\mathrm{PhCH}_{2}\right), 50.4$ (1 C, piperidyl-4-yl), 51.5 (2 C, piperidyl-2-yl, piperidyl-6-yl), $68.2\left(1 \mathrm{C},(\mathrm{Ph}) \mathrm{CH}_{2} \mathrm{O}\right), 109.2$ (1 C, C-5), 112.7 (1 C, Ph), 115.6 (1 C, Ph), 121.4 (1 C, Ph), 126.2 (2 C, Ph), 127.3 (1 C, Ph), 127.8 (1 C, Ph), 129.7 (1 C, Ph), 130.4 (1 C, Ph), 133.1 (1 C, Ph), 137.5 (1 C, C-6), 139.7 (1 C, Ph), 150,8 (1 C, C-2), 158.3 (1 C, Ph), 163.7 (1 C, C-4), C $\left(\mathrm{CH}_{2} \mathrm{~N}\right)$ could not be observed. HRMS (ESI): $m / z$ [M + H] $]^{+}$Calcd. for $\left[\mathrm{C}_{25} \mathrm{H}_{28} \mathrm{ClN}_{3} \mathrm{O}_{3}+\mathrm{H}\right]^{+} 454.1892$, found 454.1902.

1-(1-(3-((3,4-Dichlorobenzyl)oxy)phenethyl)piperidin-4-yl)-5-methylpyrimidine-2,4(1H,3H)-dione (21p). Following the general procedure $\mathrm{B}, \mathbf{1 5 c}(90 \mathrm{mg}, 0.30 \mathrm{mmol}), 28(0.10 \mathrm{~g}, 0.30 \mathrm{mmol})$ and sodium triacetoxyborohydride $(0.13 \mathrm{~g}, 0.61 \mathrm{mmol})$ in dichloroethane $(10 \mathrm{~mL})$ afforded the BOM-protected intermediate, which was deprotected with TFA $(5.0 \mathrm{~mL})$ in the presence of triethylsilane $(5.0 \mathrm{~mL})$ at $73{ }^{\circ} \mathrm{C}$ for $4 \mathrm{~h}$ to give the $21 \mathrm{p}$ (eluent system: $5 \% \mathrm{MeOH}$ in DCM, $55 \mathrm{mg}, 0.11 \mathrm{mmol}, 37 \%$ yield). ${ }^{1} \mathrm{H}$ NMR $\left(400 \mathrm{MHz}, \mathrm{DMSO}-\mathrm{d}_{6}\right) \delta$ ppm $1.79\left(\mathrm{~s}, 3 \mathrm{H}, 5-\mathrm{CH}_{3}\right), 1.92-2.06$ (m, $2 \mathrm{H}$, piperdyl-3a-yl, piperidyl-5a-yl), 2.08-2.24 (m, $2 \mathrm{H}$, piperdyl-3b-yl, piperidyl-5b-yl), 2.85-3.02 (m, $\left.2 \mathrm{H}, \mathrm{PhCH}_{2}\right), 3.06-3.22(\mathrm{~m}, 2$ $\mathrm{H}$, piperidyl-2a-yl, piperidyl-6a-yl), 3.24-3.34 (m, $\left.2 \mathrm{H}, \mathrm{CH}_{2} \mathrm{~N}\right), 3.65$ (br. s., $2 \mathrm{H}$, piperidyl-2b-yl, piperidyl-6b-yl), 4.45-4.59 (m, $1 \mathrm{H}$, piperidyl-4-yl), $5.13\left(\mathrm{~s}, 2 \mathrm{H},(\mathrm{Ph}) \mathrm{CH}_{2} \mathrm{O}\right), 6.85-6.95(\mathrm{~m}, 2 \mathrm{H}, \mathrm{Ph})$, 6.98 (br. s., 1 H, Ph), 7.28 (t, J = 7.9 Hz, 1 H, Ph), 7.38-7.48 (m, 2 H, H-6, Ph), 7.68 (d, J = 8.3 Hz, 1 H, $\mathrm{Ph}), 7.73(\mathrm{~d}, J=1.5 \mathrm{~Hz}, 1 \mathrm{H}, \mathrm{Ph}), 11.33(\mathrm{~s}, 1 \mathrm{H}, \mathrm{NH}) .{ }^{13} \mathrm{C}$ NMR $\left(101 \mathrm{MHz}, \mathrm{DMSO}-\mathrm{d}_{6}\right) \delta \mathrm{ppm} 12.2(1 \mathrm{C}$, 5- $\left.\mathrm{CH}_{3}\right), 27.2$ (2 C, piperdyl-3-yl, piperidyl-5-yl), 29.7 (1 C, $\left.\mathrm{PhCH}_{2}\right), 50.6$ (1 C, piperidyl-4-yl), 51.2 (2 C, piperidyl-2-yl, piperidyl-6-yl), $56.5\left(1 \mathrm{C}, \mathrm{CH}_{2} \mathrm{~N}\right), 67.5\left(1 \mathrm{C},(\mathrm{Ph}) \mathrm{CH}_{2} \mathrm{O}\right), 109.3(1 \mathrm{C}, \mathrm{C}-5), 112.8(1 \mathrm{C}, \mathrm{Ph})$, 115.5 (1 C, Ph), 121.5 (1 C, Ph), 127.8 (1 C, Ph), 129.4 (1 C, Ph), 129.8 (1 C, Ph), 130.4 (1 C, Ph), 130.7 (1 C, 
Ph), 131.1 (1 C, Ph), 137.4 (1 C, C-6), 138.3 (2 C, Ph), 150.7 (1 C, C-2), 158.2 (1 C, Ph), 163.7 (1 C, C-4). HRMS (ESI): $m / z[\mathrm{M}+\mathrm{H}]^{+}$Calcd. for $\left[\mathrm{C}_{25} \mathrm{H}_{27} \mathrm{Cl}_{2} \mathrm{~N}_{3} \mathrm{O}_{3}+\mathrm{H}\right]^{+} 488.1502$, found 488.1518 .

1-(1-(3-((3-Chlorobenzyl)oxy)phenethyl)piperidin-4-yl)-5-methylpyrimidine-2,4(1H,3H)-dione (21q). Following the general procedure $B, \mathbf{1 5 d}(79 \mathrm{mg}, 0.30 \mathrm{mmol}), \mathbf{2 8}(0.10 \mathrm{~g}, 0.30 \mathrm{mmol})$ and sodium triacetoxyborohydride $(0.13 \mathrm{~g}, 0.61 \mathrm{mmol})$ in dichloroethane $(10 \mathrm{~mL})$ afforded the BOM-protected intermediate, which was deprotected with TFA $(5.0 \mathrm{~mL})$ in the presence of triethylsilane $(5.0 \mathrm{~mL})$ at $73{ }^{\circ} \mathrm{C}$ for $4 \mathrm{~h}$ to give the $\mathbf{2 1 q}$ (eluent system: $5 \% \mathrm{MeOH}$ in DCM, $38 \mathrm{mg}, 0.084 \mathrm{mmol}, 28 \%$ yield). ${ }^{1} \mathrm{H}$ NMR (400 MHz, DMSO- $\mathrm{d}_{6}$ ) $\delta$ ppm 1.78 (s, $5 \mathrm{H}, 5-\mathrm{CH}_{3}$, piperdyl-3a-yl, piperidyl-5a-yl), 2.00 (br. s., $2 \mathrm{H}$, piperdyl-3b-yl, piperidyl-5b-yl), 2.84 (br. s., $\left.2 \mathrm{H}, \mathrm{PhCH}_{2}\right), 4.31-4.46\left(\mathrm{~m}, 1 \mathrm{H}\right.$, piperidyl-4-yl), $5.12\left(\mathrm{~s}, 2 \mathrm{H},\left(\mathrm{Ph}^{2} \mathrm{CH}_{2} \mathrm{O}\right), 6.87(\mathrm{t}, J=8.3 \mathrm{~Hz}, 2\right.$ $\mathrm{H}, \mathrm{Ph}$ ), 6.94 (br.s., $1 \mathrm{H}, \mathrm{Ph}), 7.23(\mathrm{t}, J=7.8 \mathrm{~Hz}, 1 \mathrm{H}, \mathrm{Ph}), 7.37-7.47$ (m, $3 \mathrm{H}, \mathrm{Ph}), 7.50-7.59$ (m, $2 \mathrm{H}, \mathrm{Ph}$, $\mathrm{H}-6), 11.26(\mathrm{~s}, 1 \mathrm{H}, \mathrm{NH}), 2 \mathrm{H}\left(\mathrm{CH}_{2} \mathrm{~N}\right)$ and $4 \mathrm{H}$ (piperidyl-2-yl, piperidyl-6-yl) could not be observed. ${ }^{13} \mathrm{C}$ NMR (101 MHz, DMSO-d 6 ) Sppm 12.1 (1 C, 5- $\mathrm{CH}_{3}$ ), 28.6 (2 C, piperdyl-3-yl, piperidyl-5-yl), 31.3 (1 C, $\mathrm{PhCH}_{2}$ ), 51.5 (1 C, piperidyl-4-yl), 51.7 (2 C, piperidyl-2-yl, piperidyl-6-yl), 68.1 (1 C, $\left(\mathrm{Ph}^{2} \mathrm{CH}_{2} \mathrm{O}\right), 109.0$ (1 C, C-5), 112.5 (1 C, Ph), 115.4 (1 C, Ph), 121.3 (1 C, Ph), 126.1 (1 C, Ph), 127.3 (1 C, Ph), 127.7 (1 C, Ph), 129.5 (1 C, Ph), 130.4 (1 C, Ph), 133.1 (1 C, Ph), 137.5 (1 C, C-6), 139.7 (1 C, Ph), 150.8 (1 C, C-2), 158.2 (1 C, $\mathrm{Ph}), 163.7(1 \mathrm{C}, \mathrm{C}-4), \mathrm{C}\left(\mathrm{CH}_{2} \mathrm{~N}\right)$ and $\mathrm{C}(\mathrm{Ph})$ could not be observed. HRMS (ESI): $\mathrm{m} / \mathrm{z}[\mathrm{M}+\mathrm{H}]^{+} \mathrm{Calcd}$. for $\left[\mathrm{C}_{25} \mathrm{H}_{28} \mathrm{ClN}_{3} \mathrm{O}_{3}+\mathrm{H}\right]^{+}$454.1892, found 454.1899.

5-Methyl-1-(1-(3-phenoxyphenethyl)piperidin-3-yl)pyrimidine-2,4(1H,3H)-dione (23). Following the general procedure $B, \mathbf{1 2 b}(65 \mathrm{mg}, 0.30 \mathrm{mmol}), 29(0.10 \mathrm{~g}, 0.30 \mathrm{mmol})$ and sodium triacetoxyborohydride $(0.13 \mathrm{~g}$, $0.61 \mathrm{mmol})$ in dichloroethane $(10 \mathrm{~mL})$ afforded the BOM-protected intermediate, which was deprotected with TFA $(5.0 \mathrm{~mL})$ in the presence of triethylsilane $(5.0 \mathrm{~mL})$ at $73^{\circ} \mathrm{C}$ for $4 \mathrm{~h}$ to give the 23 (eluent system: $5 \% \mathrm{MeOH}$ in DCM, $57 \mathrm{mg}, 0.14 \mathrm{mmol}, 46 \%$ yield). ${ }^{1} \mathrm{H}$ NMR $\left(400 \mathrm{MHz}, \mathrm{DMSO}-\mathrm{d}_{6}\right) \delta \mathrm{ppm} 1.43-1.56(\mathrm{~m}$, $1 \mathrm{H}$, piperdyl-5a-yl), 1.59-1.80 (m, $6 \mathrm{H}, 5-\mathrm{CH}_{3}$, piperdyl-4-yl, piperdyl-5b-yl), $2.07(\mathrm{t}, J=12.9 \mathrm{~Hz}, 1 \mathrm{H}$, piperdyl-6a-yl), $2.22\left(\mathrm{t}, J=10.3 \mathrm{~Hz}, 1 \mathrm{H}\right.$, piperdyl-2a-yl), $2.56\left(\mathrm{t}, J=4.6 \mathrm{~Hz}, 2 \mathrm{H}, \mathrm{CH}_{2} \mathrm{~N}\right), 2.68-2.81(\mathrm{~m}$, $3 \mathrm{H}$, piperdyl-6b-yl, $\left.\mathrm{PhCH}_{2}\right), 2.82-2.90(\mathrm{~m}, 1 \mathrm{H}$, piperdyl-2b-yl), 4.31-4.43 (m, $1 \mathrm{H}$, piperdyl-3-yl), 6.81 $(\mathrm{dd}, J=8.1,1.8 \mathrm{~Hz}, 1 \mathrm{H}, \mathrm{Ph}), 6.90(\mathrm{~s}, 1 \mathrm{H}, \mathrm{Ph}), 6.95-7.04(\mathrm{~m}, 3 \mathrm{H}, \mathrm{Ph}), 7.12(\mathrm{t}, J=7.6 \mathrm{~Hz}, 1 \mathrm{H}, \mathrm{Ph}), 7.28(\mathrm{t}$, $J=7.8 \mathrm{~Hz}, 1 \mathrm{H}, \mathrm{Ph}), 7.38(\mathrm{t}, J=7.9 \mathrm{~Hz}, 2 \mathrm{H}, \mathrm{Ph}), 7.68(\mathrm{~s}, 1 \mathrm{H}, \mathrm{H}-6), 11.23(\mathrm{~s}, 1 \mathrm{H}, \mathrm{NH}) .{ }^{13} \mathrm{C} \mathrm{NMR}(101$ MHz, DMSO-d $\left.{ }_{6}\right) \delta$ ppm $12.0\left(1 \mathrm{C}, 5-\mathrm{CH}_{3}\right), 23.9$ (1 C, piperdyl-5-yl), 28.1 (1 C, piperdyl-4-yl), $32.3(1 \mathrm{C}$, $\left.\mathrm{PhCH}_{2}\right), 51.0$ (1 C, piperdyl-3-yl), 52.2 (1 C, piperdyl-6-yl), $56.5\left(1 \mathrm{C}\right.$, piperdyl-2-yl), $59.2\left(1 \mathrm{C} \mathrm{CH}_{2} \mathrm{~N}\right)$, 108.6 (1 C, C-5), 116.1 (1 C, Ph), 118.5 (2 C, Ph), 119.0 (1 C, Ph), 123.3 (1 C, Ph), 123.9 (1 C, Ph), 129.7 (1 C, Ph), 130.0 (2 C, Ph), 138.0 (1 C, C-6), 142.7 (1 C, Ph), 150.8 (1 C, C-2), 156.5 (1 C, Ph), 156.7 (1 C, Ph), 163.7 (1 C, C-4). HRMS (ESI): $m / z[\mathrm{M}+\mathrm{H}]^{+}$Calcd. for $\left[\mathrm{C}_{24} \mathrm{H}_{27} \mathrm{~N}_{3} \mathrm{O}_{3}+\mathrm{H}\right]^{+}$406.2125, found 406.2125.

5-Methyl-1-(1-(2-(3-phenoxyphenyl)acetyl)piperidin-4-yl)pyrimidine-2,4(1H,3H)-dione (26). To a solution of $10 \mathrm{~b}(0.2 \mathrm{~g}, 0.82 \mathrm{mmol})$ in $\mathrm{MeOH}(5.0 \mathrm{~mL})$ was added $1 \mathrm{M} \mathrm{NaOH}(5.0 \mathrm{~mL})$, the resulting reaction mixture was stirred at $50{ }^{\circ} \mathrm{C}$ for $2 \mathrm{~h}$. After cooling to room temperature, the reaction mixture was treated with $1 \mathrm{~N}$ aq. $\mathrm{HCl}$ to $\mathrm{pH} 2-3$. The generated white precipitate was collected through filtration and dried in vacuo. The intermediate $(0.17 \mathrm{~g}, 0.74 \mathrm{mmol}), 28(0.26 \mathrm{~g}, 0.79 \mathrm{mmol}), \mathrm{EDC} \mathrm{HCl}(0.29 \mathrm{~g}$, $1.5 \mathrm{mmol})$ and 4-dimethylaminopyridine (DMAP) $(9.1 \mathrm{mg}, 7.4 \mu \mathrm{mol})$ were dissolved in DCM $(30 \mathrm{~mL})$, and the reaction mixture was stirred at room temperature for overnight to afford BOM protected intermediate, which was deprotected with $\mathrm{Pd} / \mathrm{C}(10 \%), \mathrm{H}_{2}$ and $\mathrm{HCOOH}(0.5 \%)$ in i-propanol/ $\mathrm{H}_{2} \mathrm{O}$ (10/1 mL) [30] to give 26 (eluent system: $5 \% \mathrm{MeOH}$ in DCM, $45 \mathrm{mg}, 0.11 \mathrm{mmol}, 13 \%$ yield). ${ }^{1} \mathrm{H}$ NMR (300 MHz, DMSO-d 6 ) $\delta$ ppm 1.54-1.83 (m, 7 H, 5- $\mathrm{CH}_{3}$, piperdyl-3-yl, piperidyl-5-yl), 2.53-2.67 (m, $1 \mathrm{H}$, piperidyl-2/6-yl), $3.07\left(\mathrm{t}, J=10.8 \mathrm{~Hz}, 1 \mathrm{H}\right.$, piperidyl-2/6-yl), 3.59-3.85 (m, $\left.2 \mathrm{H}, \mathrm{COCH}_{2}\right), 4.05(\mathrm{~d}$, $J=13.5 \mathrm{~Hz}, 1 \mathrm{H}$, piperidyl-2/6-yl), 4.43-4.56 (m, $2 \mathrm{H}$, piperidyl-4-yl, piperidyl-2/6-yl), 6.82-6.91 (m, $2 \mathrm{H}, \mathrm{Ph}), 6.95-7.03$ (m, 3 H, Ph), 7.08-7.15 (m, $1 \mathrm{H}, \mathrm{Ph}), 7.31$ (t, J = 7.8 Hz, $1 \mathrm{H}, \mathrm{Ph}), 7.34-7.41$ (m, 2 $\mathrm{H}, \mathrm{Ph}), 7.53(\mathrm{~d}, J=0.9 \mathrm{~Hz}, 1 \mathrm{H}, \mathrm{H}-6), 11.20(\mathrm{~s}, 1 \mathrm{H}, \mathrm{NH}) .{ }^{13} \mathrm{C}$ NMR $\left(101 \mathrm{MHz}, \mathrm{DMSO}-\mathrm{d}_{6}\right) \delta \mathrm{ppm} 12.1$ (1 C, 5- $\left.\mathrm{CH}_{3}\right), 29.7$ (1 C, piperidyl-3/5-yl), 30.5 (1 C, piperidyl-3/5-yl), $38.9\left(1 \mathrm{C},\left(\mathrm{CO}^{2} \mathrm{CH}_{2}\right), 40.8(1 \mathrm{C}\right.$, piperidyl-2/6-yl), 44.7 (1 C, piperidyl-2/6-yl), 52.1 (1 C, piperidyl-4-yl), 109.1 (1 C, C-5), 116.7 (1 C, Ph), 118.7 (2 C, Ph), 119.5 (1 C, Ph), 123.5 (1 C, Ph), 124.4 (1 C, Ph), 129.9 (1 C, Ph), 130.1 (2 C, Ph), 137.7 
(1 C, Ph), 138.2 (1 C, C-6), 150.8 (1 C, C-2), 156.6 (1 C, Ph), 156.7 (1 C, Ph), 163.8 (1 C, C-4), 168.5 (1 C, $\left.\mathrm{CO}\left(\mathrm{CH}_{2}\right)\right)$. HRMS (ESI): $\mathrm{m} / \mathrm{z}[\mathrm{M}+\mathrm{H}]^{+}$Calcd. for $\left[\mathrm{C}_{24} \mathrm{H}_{25} \mathrm{~N}_{3} \mathrm{O}_{4}+\mathrm{H}\right]^{+} 420.1918$, found 420.1925 .

1-(3-Phenoxyphenethyl)-4-phenylpiperidine (27). To a solution of $\mathbf{1 2 b}(0.12 \mathrm{~g}, 0.56 \mathrm{mmol})$ and 4-phenylpiperidine $(0.14 \mathrm{~g}, 0.87 \mathrm{mmol})$ in dichloroethane $(10 \mathrm{~mL})$ was added sodium triacetoxyborohydride $(0.24 \mathrm{~g}, 1.1 \mathrm{mmol})$, the resulting mixture was stirred at room temperature for overnight. The reaction mixture was diluted with $\mathrm{DCM}$, and extracted with sat. $\mathrm{NaHCO}_{3}$ and brine. The collected organic layer was dried, concentrated and purified to give 27 (58 mg, $0.16 \mathrm{mmol}, 29 \%$ yield). ${ }^{1} \mathrm{H}$ NMR (300 MHz, CDCl 3 ) $\delta$ ppm 1.91 (br. s., 4 H, piperdyl-3-yl, piperidyl-5-yl), 2.12-2.31 (m, $2 \mathrm{H}$, piperidyl-2a-yl, piperidyl-6a-yl), 2.46-2.62 (m, $1 \mathrm{H}$, piperidyl-4-yl), 2.65-2.77 $\left(\mathrm{m}, 2 \mathrm{H}, \mathrm{CH}_{2} \mathrm{~N}\right)$, 2.83-2.97 (m, $\left.2 \mathrm{H}, \mathrm{PhCH}_{2}\right), 3.20$ (d, J = 10.8 Hz, $2 \mathrm{H}$, piperidyl-2b-yl, piperidyl-6b-yl), 6.82 - 6.95 (m, 2 $\mathrm{H}, \mathrm{Ph}), 6.96-7.07(\mathrm{~m}, 3 \mathrm{H}, \mathrm{Ph}), 7.12(\mathrm{t}, J=7.3 \mathrm{~Hz}, 1 \mathrm{H}, \mathrm{Ph}), 7.17-7.46(\mathrm{~m}, 8 \mathrm{H}, \mathrm{Ph}) .{ }^{13} \mathrm{C}$ NMR $(75 \mathrm{MHz}$, $\left.\mathrm{CDCl}_{3}\right)$ Sppm 33.0 (2 C, piperdyl-3-yl, piperidyl-5-1), $33.2\left(1 \mathrm{C}, \mathrm{PhCH}_{2}\right), 42.4(1 \mathrm{C}$, piperidyl-4-yl), 54.1 (2 C, piperidyl-2-yl, piperidyl-6-yl), $60.3\left(1 \mathrm{C}, \mathrm{CH}_{2} \mathrm{~N}\right), 116.5(1 \mathrm{C}, \mathrm{Ph}), 118.8(1 \mathrm{C}, \mathrm{Ph}), 119.1$ (1 C, Ph), 123.2 (1 C, Ph), 123.6 (1 C, Ph), 126.2 (1 C, Ph), 126.8 (3 C, Ph), 128.4 (2 C, Ph), 129.6 (1 C, Ph), 129.7 (2 C, Ph), 142.1 (1 C, Ph), 145.9 (1 C, Ph), 157.1 (1 C, Ph), 157.8 (1 C, Ph). HRMS (ESI): m/z [M + $\mathrm{H}]^{+}$Calcd. for $\left[\mathrm{C}_{25} \mathrm{H}_{27} \mathrm{NO}+\mathrm{H}\right]^{+} 358.2166$, found 358.2164 .

\section{Conclusions}

Starting from the earlier reported compound 3, 19 analogs were synthesized and evaluated for the inhibitory potencies of both MtbTMPK and M. tuberculosis (H37Rv) in vitro growths. Selected substituents on the terminal phenyl ring slightly improved the inhibitory potency. Surprisingly, the 3-Cl analog (21j) was three-fold more potent than compound $\mathbf{3}$ as the MtbTMPK inhibitor. Substitution of the distal phenyl ring of $\mathbf{3}$ and $\mathbf{2 1 n}$ afforded analogs with superior whole cell antimycobacterial activity compared to 3 . These results provide possible directions for further investigations of $M t b T M P K$ inhibitors as antituberculosis agents.

Supplementary Materials: The following are available online at http://www.mdpi.com/1420-3049/25/12/2805/s1, Figures S1-S43: NMR spectra of final compounds.

Author Contributions: Chemistry and writing-original draft preparation, Y.J.; conceptualization, S.V.C.; methodology, H.E.F., G.C., H.M.-L. and H.I.M.B.; writing-review and editing, F.H., H.M.-L., H.I.M.B. and S.V.C. and supervision, S.V.C. and M.D.P.R. All authors discussed the results and contributed to the final manuscript. All authors have read and agreed to the published version of the manuscript.

Funding: This research was funded by the China Scholarship Council (grant number 201607060021) and, in part, by the Intramural Research Program of the NIAID, NIH. S.V.C. thanks the Hercules Foundation (project AUGE/17/22 "Pharm-NMR").

Conflicts of Interest: The authors declare no conflict of interest.

\section{References}

1. WHO. Global Tuberculosis Report 2019; World Health Organization: Geneva, Switzerland, 2019.

2. Uplekar, M.; Weil, D.; Lonnroth, K.; Jaramillo, E.; Lienhardt, C.; Dias, H.M.; Falzon, D.; Floyd, K.; Gargioni, G.; Getahun, H.; et al. WHO's new end TB strategy. Lancet 2015, 385, 1799-1801. [CrossRef]

3. Lange, C.; Dheda, K.; Chesov, D.; Mandalakas, A.M.; Udwadia, Z.; Horsburgh, C.R., Jr. Management of drug-resistant tuberculosis. Lancet 2019, 394, 953-966. [CrossRef]

4. Karabanovich, G.; Dusek, J.; Savkova, K.; Pavlis, O.; Pavkova, I.; Korabecny, J.; Kucera, T.; Kocova Vlckova, H.; Huszar, S.; Konyarikova, Z.; et al. Development of 3,5-Dinitrophenyl-Containing 1,2,4-Triazoles and Their Trifluoromethyl Analogues as Highly Efficient Antitubercular Agents Inhibiting Decaprenylphosphoryl-beta-d-ribofuranose 2'-Oxidase. J. Med. Chem. 2019, 62, 8115-8139. [CrossRef] [PubMed]

5. WHO. WHO Consolidated Guidelines on Drug-Resistant Tuberculosis Treatment; World Health Organization: Geneva, Switzerland, 2019. 
6. Hoffmann, H.; Kohl, T.A.; Hofmann-Thiel, S.; Merker, M.; Beckert, P.; Jaton, K.; Nedialkova, L.; Sahalchyk, E.; Rothe, T.; Keller, P.M.; et al. Delamanid and Bedaquiline Resistance in Mycobacterium tuberculosis Ancestral Beijing Genotype Causing Extensively Drug-Resistant Tuberculosis in a Tibetan Refugee. Am. J. Respir. Crit. Care Med. 2016, 193, 337-340. [CrossRef]

7. Haver, H.L.; Chua, A.; Ghode, P.; Lakshminarayana, S.B.; Singhal, A.; Mathema, B.; Wintjens, R.; Bifani, P. Mutations in genes for the F420 biosynthetic pathway and a nitroreductase enzyme are the primary resistance determinants in spontaneous in vitro-selected PA-824-resistant mutants of Mycobacterium tuberculosis. Antimicrob. Agents Chemother. 2015, 59, 5316-5323. [CrossRef]

8. Food and Drug Administration. FDA Approved Drug Products. Available online: https://www.accessdata. fda.gov/scripts/cder/daf/index.cfm?event=overview.process\&ApplNo=212862 (accessed on 14 August 2019).

9. Jong, A.Y.; Kuo, C.L.; Campbell, J.L. The CDC8 gene of yeast encodes thymidylate kinase. J. Biol. Chem. 1984, 259, 11052-11059.

10. Cole, S.T.; Brosch, R.; Parkhill, J.; Garnier, T.; Churcher, C.; Harris, D.; Gordon, S.V.; Eiglmeier, K.; Gas, S.; Barry, C.E.; et al. Deciphering the biology of Mycobacterium tuberculosis from the complete genome sequence. Nature 1998, 393, 537-544. [CrossRef]

11. Fioravanti, E.; Haouz, A.; Ursby, T.; Munier-Lehmann, H.; Delarue, M.; Bourgeois, D. Mycobacterium tuberculosis thymidylate kinase: Structural studies of intermediates along the reaction pathway. J. Mol. Biol. 2003, 327, 1077-1092. [CrossRef]

12. Li de la Sierra, I.; Munier-Lehmann, H.; Gilles, A.M.; Barzu, O.; Delarue, M. X-ray structure of TMP kinase from Mycobacterium tuberculosis complexed with TMP at 1.95 A resolution. J. Mol. Biol. 2001, 311, 87-100. [CrossRef]

13. Fioravanti, E.; Adam, V.; Munier-Lehmann, H.; Bourgeois, D. The crystal structure of Mycobacterium tuberculosis thymidylate kinase in complex with 3'-azidodeoxythymidine monophosphate suggests a mechanism for competitive inhibition. Biochemistry 2005, 44, 130-137. [CrossRef]

14. Song, L.; Merceron, R.; Gracia, B.; Quintana, A.L.; Risseeuw, M.D.P.; Hulpia, F.; Cos, P.; Ainsa, J.A.; Munier-Lehmann, H.; Savvides, S.N.; et al. Structure Guided Lead Generation toward Nonchiral, M. tuberculosis Thymidylate Kinase Inhibitors. J. Med. Chem. 2018, 61, 2753-2775. [CrossRef]

15. Naik, M.; Raichurkar, A.; Bandodkar, B.S.; Varun, B.V.; Bhat, S.; Kalkhambkar, R.; Murugan, K.; Menon, R.; Bhat, J.; Paul, B.; et al. Structure guided lead generation for M. tuberculosis thymidylate kinase (Mtb TMK): Discovery of 3-cyanopyridone and 1,6-naphthyridin-2-one as potent inhibitors. J. Med. Chem. 2015, 58, 753-766. [CrossRef]

16. Haouz, A.; Vanheusden, V.; Munier-Lehmann, H.; Froeyen, M.; Herdewijn, P.; Van Calenbergh, S.; Delarue, M. Enzymatic and structural analysis of inhibitors designed against Mycobacterium tuberculosis thymidylate kinase. New insights into the phosphoryl transfer mechanism. J. Biol. Chem. 2003, 278, 4963-4971. [CrossRef]

17. Vanheusden, V.; Van Rompaey, P.; Munier-Lehmann, H.; Pochet, S.; Herdewijn, P.; Van Calenbergh, S. Thymidine and thymidine-5'-O-monophosphate analogues as inhibitors of Mycobacterium tuberculosis thymidylate kinase. Bioorg. Med. Chem. Lett. 2003, 13, 3045-3048. [CrossRef]

18. Vanheusden, V.; Munier-Lehmann, H.; Froeyen, M.; Dugue, L.; Heyerick, A.; De Keukeleire, D.; Pochet, S.; Busson, R.; Herdewijn, P.; Van Calenbergh, S. 3'-C-branched-chain-substituted nucleosides and nucleotides as potent inhibitors of Mycobacterium tuberculosis thymidine monophosphate kinase. J. Med. Chem. 2003, 46, 3811-3821. [CrossRef]

19. Van Poecke, S.; Munier-Lehmann, H.; Helynck, O.; Froeyen, M.; Van Calenbergh, S. Synthesis and inhibitory activity of thymidine analogues targeting Mycobacterium tuberculosis thymidine monophosphate kinase. Bioorg. Med. Chem. 2011, 19, 7603-7611. [CrossRef] [PubMed]

20. Song, L.; Risseeuw, M.D.P.; Froeyen, M.; Karalic, I.; Goeman, J.; Cappoen, D.; Van der Eycken, J.; Cos, P.; Munier-Lehmann, H.; Van Calenbergh, S. Elaboration of a proprietary thymidylate kinase inhibitor motif towards anti-tuberculosis agents. Bioorg. Med. Chem. 2016, 24, 5172-5182. [CrossRef] [PubMed]

21. Jian, Y.; Risseeuw, M.D.P.; Froeyen, M.; Song, L.; Cappoen, D.; Cos, P.; Munier-Lehmann, H.; van Calenbergh, S. 1-(Piperidin-3-yl)thymine amides as inhibitors of M. tuberculosis thymidylate kinase. J. Enzym. Inhib. Med. Chem. 2019, 34, 1730-1739. [CrossRef] [PubMed]

22. Martinez-Botella, G.; Breen, J.N.; Duffy, J.E.; Dumas, J.; Geng, B.; Gowers, I.K.; Green, O.M.; Guler, S.; Hentemann, M.F.; Hernandez-Juan, F.A.; et al. Discovery of selective and potent inhibitors of gram-positive bacterial thymidylate kinase (TMK). J. Med. Chem. 2012, 55, 10010-10021. [CrossRef] 
23. Zhu, Z.; Chen, H.-G.; Goel, O.P.; Chan, O.H.; Stilgenbauer, L.A.; Stewart, B.H. Phosphate prodrugs of PD154075. Bioorg. Med. Chem. Lett. 2000, 10, 1121-1124. [CrossRef]

24. Elgaher, W.A.M.; Fruth, M.; Groh, M.; Haupenthal, J.; Hartmann, R.W. Expanding the scaffold for bacterial RNA polymerase inhibitors: Design, synthesis and structure-activity relationships of ureido-heterocyclic-carboxylic acids. RSC Adv. 2014, 4, 2177-2194. [CrossRef]

25. Elgaher, W.A.M.; Sharma, K.K.; Haupenthal, J.; Saladini, F.; Pires, M.; Real, E.; Mely, Y.; Hartmann, R.W. Discovery and Structure-Based Optimization of 2-Ureidothiophene-3-carboxylic Acids as Dual Bacterial RNA Polymerase and Viral Reverse Transcriptase Inhibitors. J. Med. Chem. 2016, 59, 7212-7222. [CrossRef] [PubMed]

26. Meanwell, N.A.; Krystal, M.R.; Nowicka-Sans, B.; Langley, D.R.; Conlon, D.A.; Eastgate, M.D.; Grasela, D.M.; Timmins, P.; Wang, T.; Kadow, J.F. Inhibitors of HIV-1 Attachment: The Discovery and Development of Temsavir and its Prodrug Fostemsavir. J. Med. Chem. 2018, 61, 62-80. [CrossRef] [PubMed]

27. Hrdlicka, P.J.; Jepsen, J.S.; Wengel, J. Synthesis and Biological Evaluation of Conformationally Restricted and Nucleobase Modified Analogs of the Anticancer Compound 3'-C-Ethynylcytidine (ECyd). Nucleosides Nucleotides Nucleic Acids 2005, 24, 397-400. [CrossRef]

28. Jean-Yves, M.; Benoit, J. Synthesis and Reactivity of 7-Azaindoles (1H-Pyrrolo(2,3-b)pyridine). Curr. Org. Chem. 2001, 5, 471-506.

29. Mehta, A.; Jaouhari, R.; Benson, T.J.; Douglas, K.T. Improved Efficiency and Selectivity in Peptide-Synthesis-Use of Triethylsilane as a Carbocation Scavenger in Deprotection of Tert-Butyl Esters and Tert-Butoxycarbonyl-Protected Sites. Tetrahedron Lett. 1992, 33, 5441-5444. [CrossRef]

30. Aleiwi, B.A.; Kurosu, M. A reliable Pd-mediated hydrogenolytic deprotection of BOM group of uridine ureido nitrogen. Tetrahedron Lett. 2012, 53, 3758-3762. [CrossRef]

31. Laskowski, R.A.; Swindells, M.B. LigPlot+: Multiple Ligand-Protein Interaction Diagrams for Drug Discovery. J. Chem. Inf. Model. 2011, 51, 2778-2786. [CrossRef]

32. Pettersen, E.F.; Goddard, T.D.; Huang, C.C.; Couch, G.S.; Greenblatt, D.M.; Meng, E.C.; Ferrin, T.E. UCSF chimera - A visualization system for exploratory research and analysis. J. Comput. Chem. 2004, 25, 1605-1612. [CrossRef]

33. Munier-Lehmann, H.; Chaffotte, A.; Pochet, S.; Labesse, G. Thymidylate kinase of Mycobacterium tuberculosis: A chimera sharing properties common to eukaryotic and bacterial enzymes. Protein Sci. 2001, 10, 1195-1205. [CrossRef]

34. Blondin, C.; Serina, L.; Wiesmuller, L.; Gilles, A.M.; Barzu, O. Improved spectrophotometric assay of nucleoside monophosphate kinase activity using the pyruvate kinase/lactate dehydrogenase coupling system. Anal. Biochem. 1994, 220, 219-221. [CrossRef] [PubMed]

35. Morris, G.M.; Huey, R.; Lindstrom, W.; Sanner, M.F.; Belew, R.K.; Goodsell, D.S.; Olson, A.J. AutoDock4 and AutoDockTools4: Automated docking with selective receptor flexibility. J. Comput. Chem. 2009, 30, 2785-2791. [CrossRef] [PubMed]

36. Park, Y.; Pacitto, A.; Bayliss, T.; Cleghorn, L.A.; Wang, Z.; Hartman, T.; Arora, K.; Ioerger, T.R.; Sacchettini, J.; Rizzi, M.; et al. Essential but Not Vulnerable: Indazole Sulfonamides Targeting Inosine Monophosphate Dehydrogenase as Potential Leads against Mycobacterium tuberculosis. ACS Infect. Dis. 2017, 3, 18-33. [CrossRef] [PubMed]

Sample Availability: Samples of the compounds 21a-21q, 23, 26 and 27 are available from the authors. 